\title{
Global Transcriptomic Changes Induced by Infection of Cucumber (Cucumis sativus L.) with Mild and Severe Variants of Hop Stunt Viroid
}

\author{
Changjian Xia ${ }^{1,2}$, Shifang $\mathrm{Li}^{1}$, Wanying Hou ${ }^{3}$, Zaifeng Fan ${ }^{2}$, Hong Xiao ${ }^{1}$, Meiguang Lu ${ }^{1}$, \\ Teruo Sano ${ }^{4 *}$ and Zhixiang Zhang ${ }^{1 *}$ \\ ${ }^{1}$ State Key Laboratory for Biology of Plant Diseases and Insect Pests, Institute of Plant Protection, Chinese Academy of \\ Agricultural Sciences, Beijing, China, ${ }^{2}$ State Key Laboratory for Agro-Biotechnology, Key Laboratory of Pest Monitoring and \\ Green Management, Department of Plant Pathology, College of Plant Protection, China Agricultural University, Beijing, China, \\ ${ }^{3}$ Key Laboratory of Tobacco Pest Monitoring Controlling and Integrated Management, State Tobacco Monopoly Bureau, \\ Institue of Tobacco Research, Chinese Academy of Agricultural Sciences, Qingdao, China, ${ }^{4}$ Faculty of Agriculture and Life \\ Science, Hirosaki University, Hirosaki, Japan
}

OPEN ACCESS

Edited by:

Ricardo Flores,

Instituto de Biología Molecular y Celular de Plantas (CSIC), Spain

Reviewed by:

Bishwo N. Adhikari, Agricultural Research Service (USDA), United States

Selma Gago-Zachert, Leibniz-Institut für Pflanzenbiochemie (IPB), Germany

*Correspondence: Teruo Sano sano@hirosaki-u.ac.jp Zhixiang Zhang zxzhang@ippcaas.cn

\section{Specialty section: \\ This article was submitted to \\ Virology, \\ a section of the journal \\ Frontiers in Microbiology}

Received: 09 August 2017 Accepted: 23 November 2017 Published: 12 December 2017

Citation:

Xia CJ, Li SF, Hou WY, Fan ZF, Xiao H, Lu MG, Sano T and Zhang ZX (2017) Global Transcriptomic Changes Induced by Infection of Cucumber (Cucumis sativus L.) with Mild and Severe Variants of Hop Stunt Viroid. Front. Microbiol. 8:2427. doi: 10.3389/fmicb.2017.02427
Fifteen years after transfer to hops, hop stunt viroid-grapevine (HSVd-g) was replaced by HSVd-hop (HSVd-h), a sequence variant that contains changes at five different positions. HSVd-g54 is a laboratory mutant derived from HSVd-g that differs from its progenitor by a single $\mathrm{G}$ to A substitution at position 54. While infection by HSVd-h induces only mild stunting in cucumber (Cucumis sativus L.), HSVd-g54 induces much more severe symptoms in this indicator host. Comparison of transcriptome profiles of cucumber infected with HSVd-h or HSVd-g54 with those of mock-inoculated controls obtained by whole transcriptome shotgun sequencing revealed that many genes related to photosynthesis were down-regulated following infection. In contrast, genes encoding RNA-dependent RNA polymerase 1 (CsRDR1), especially CsRDR1c1 and CsRDR1c2, as well as those related to basal defense responses were up-regulated. Expression of genes associated with phytohormone signaling pathways were also altered, indicating that viroid infection initiates a complex array of changes in the host transcriptome. HSVd-g54 induced an earlier and stronger response than HSVd-h, and further examination of these differences will contribute to a better understanding of the mechanisms that determine viroid pathogenicity.

Keywords: hop stunt viroid, cucumber, RNA-seq, RNA-dependent RNA polymerase 1 (CsRDR1), salicylic acid, photosynthesis

\section{INTRODUCTION}

Viroids are small, circular, non-protein-coding infectious RNAs that have the ability to replicate autonomously when inoculated into higher plants. Their genomic RNAs range in size from 246 to 401 nucleotides (nt) (Flores et al., 2015; Daròs, 2016; Gago-Zachert, 2016), and some viroids infect economically important crops where they can cause devastating diseases. The symptoms of viroid diseases include chlorosis, epinasty, leaf deformation and necrosis, stunting, fruit distortion, and plant death (Flores et al., 2005). As demonstrated for several other types of plant pathogens (Boyko et al., 2007; Babu et al., 2008), comprehensive analysis of gene expression in viroid-infected host is critical to understanding the molecular mechanisms responsible for viroid pathogenesis. 
Published studies have used either mRNA differential display (Tessitori et al., 2007) or hybridization-based microarray technologies (Owens et al., 2012; Rizza et al., 2012) to examine the number and types of host genes that are differentially expressed in response to viroid infection. The viroid-host combination most frequently studied has been tomato plants infected with potato spindle tuber viroid (PSTVd). Among the biological functions affected by viroid infection are defense and stress responses, cell wall structure, chloroplast biogenesis, protein metabolism, peroxidase, and symporter activities (Itaya et al., 2002; Owens et al., 2012; Rizza et al., 2012). Genes involved in the biosynthesis of gibberellin, jasmonic acid, and other phytohormones also showed changes in expression during PSTVd infection (Wang et al., 2011; Owens et al., 2012). It should be noted that most of the host genes whose expression was altered by viroid infections are sensitive to many different biotic stresses, indicating that one or more shared signal transduction pathways or gene regulation networks may be involved in viroid disease induction. However, there is not enough data at present to construct a clear pathway or network to explain viroid disease progression. A recentlydeveloped large-scale RNA sequencing-based strategy (i.e., RNAseq; Wang et al., 2009) promises to change this situation.

RNA-seq is a high-throughput approach to transcriptome profiling that uses next generation sequencing (NGS) technologies for the analysis of gene expression (Wang et al., 2009; Ozsolak and Milos, 2011). RNA-seq eliminates several of the problems associated with microarray technologies, including its restriction to known genes and limited dynamic range of detection (Ozsolak and Milos, 2011; Van Verk et al., 2013). RNA-seq can generate a far more precise estimate of mRNA levels and transcript isoforms at a much lower cost. These advantages, coupled with higher reproducibility and lower RNA sample requirements (Wang et al., 2009; Van Verk et al., 2013) make RNA-seq a powerful tool for global genome expression studies. It can be used to investigate specific developmental stages or physiological conditions as well as tissues in normal or diseased states (Ozsolak and Milos, 2011; Van Verk et al., 2013).

In plant pathology, transcriptome analysis has been widely used to examine global gene expression profiles in plants infected with fungi, bacteria, or virus. However, only three such studies have been performed for viroid-infected plants. Most recently, the transcriptome of PSTVd-infected tomato (Zheng et al., 2017), hop stunt viroid (HSVd)-infected hop (Kappagantu et al., 2017) and hop latent viroid (HLVd)- and citrus bark cracking viroid (CBCVd)-infected hop (Pokorn et al., 2017) were comprehensively analyzed. Tomato genes involved in plant immune responses, mainly those in the calcium-dependent protein kinase and mitogen-activated protein kinase signaling cascades were found to be strongly up-regulated in response to PSTVd infection (Zheng et al., 2017). Hop genes involved in defense, lipid and terpenoid metabolism showed differential expression due to HSVd infection (Kappagantu et al., 2017). Many hop transcripts share nucleotide similarities with HLVdand CBCVd-derived small RNAs, so they could be silenced in an RNA interference process, and four pathogenesis related genes were highly expressed in leaves of HLVd- and CBCVd-infected hop plants (Pokorn et al., 2017). These results provide new insights into viroid pathogenesis. Comparable analyses of other viroid-host combinations should be helpful for understanding the mechanisms that underlie viroid disease induction.

In this study, we employed HSVd-infected cucumber (Cucumis sativus cv. "Suyo") as a model system in which to examine genome-wide changes in gene expression using RNAseq. Cucumber plants infected by two HSVd variants that differ in pathogenicity were analyzed at different times post inoculation. We found that the severe variant (HSVd-g54) induced an earlier and stronger response than the mild variant (HSVd-h); furthermore, HSVd infection depressed photosynthesis, disrupted phytohormone homeostasis, and possibly induced the accumulation of salicylic acid (SA). HSVd infection also triggered basal defense responses and the expression of genes coding for RNA-dependent RNA polymerase 1 (CsRDR1), specially CsRDR1c1 and CsRDR1c2. These results provide insights into the interaction between cucumber and HSVd and contribute to a better understanding of viroid pathogenesis.

\section{MATERIALS AND METHODS}

\section{HSVd Variants}

Two different variants of HSVd were used in our experiments. Both HSVd-h (AB039271) and HSVd-g54 are related to an HSVd isolate (AB219944) originally retrieved from grapevine in Japan, HSVd-g (grapevine) (Sano et al., 2001). As described by Kawaguchi-Ito et al. (2009), HSVd-h is a novel variant recovered from hop plants inoculated with HSVd-g 15 years earlier; it differs from HSVd-g at five positions. HSVd-g54, in contrast, contains only a single $\mathrm{A} / \mathrm{G}$ substitution at position 54 with respect to HSVd-g.

\section{Preparation of Inoculum}

Infectious clones of HSVd-h and HSVd-g54 were constructed by overnight incubation of unit-length cDNAs with blunt ends with T4 DNA ligase (Promega) at $4^{\circ} \mathrm{C}$ to generate dimers. Dimeric cDNAs were purified by gel electrophoresis, a dA was added to the $3^{\prime}$ end using Taq DNA polymerase (Tiangen Biotech, Beijing), and the modified dimers were ligated into the PGEM-T vector (Promega). Two recombinant plasmids containing head-to-tail tandem cDNA repeats, pG-dHSh and pG-dHSg54, were then selected by DNA sequencing.

Because the HSVd-h cDNA dimer was ligated into the vector in the forward orientation, pG-dHSh was linearized with Spe I (Takara) before in vitro transcription using T7 RNA polymerase (Promega). HSVd-g54 cDNA dimer was ligated into the vector in reverse orientation; thus, the resulting recombinant plasmid was linearized with Nco I (Takara) before transcription using SP6 RNA polymerase (Promega). Before inoculation RNA transcripts were adjusted to a final concentration of $100 \mathrm{ng} \mathrm{RNA} / \mu \mathrm{L}$ in $100 \mathrm{mM}$ sodium phosphate buffer ( $\mathrm{pH} 7.5$ ) containing $0.3 \mathrm{mg} / \mathrm{ml}$ bentonite.

\section{Plant Growth and Inoculation}

Cucumber seedlings were grown in a greenhouse with supplementary lighting ( $16 \mathrm{~h}$ per day). The temperature 
was maintained at $28^{\circ} \mathrm{C}$ in the daytime and $25^{\circ} \mathrm{C}$ at night. One-week-old seedlings with two expanded cotyledons were used for viroid inoculation. One cotyledon of each seedling was dusted with carborundum (600 mesh) and rubbed with $20 \mu \mathrm{L}$ aliquots of inoculum using a sterile cotton swab. Twelve cucumber seedlings were inoculated for each treatment (HSVd-g54, HSVd-h and mock). Inoculated plants were rinsed with distilled water, placed in darkness for $8 \mathrm{~h}$, and then kept in the greenhouse with the same environmental conditions for 4-5 weeks.

\section{RNA Extraction, RT-PCR, Cloning, and Sequencing}

Total RNAs used for northern-blot hybridization and RT-PCR detection were extracted from cucumber cotyledons (2 dpi) and top leaves (14 and $28 \mathrm{dpi}$ ) using a CTAB method as described previously (Adkar-Purushothama et al., 2015). For RNA-seq and RT-qPCR analysis, total RNAs from cotyledons (2 dpi) and top leaves (14 and $28 \mathrm{dpi}$ ) of the inoculated cucumber plants were extracted using TRNzol-A+ reagent according to the manufacturer's instructions (Tiangen Biotech, Beijing). The quality and quantity of all RNA samples were assessed by agarose gel electrophoresis and absorbance at $260 \mathrm{~nm}$ using a NanoDrop 2000 spectrophotometer (ThermoFisher Scientific). Furthermore, the integrity of the RNA preparations used for cDNA libraries for RNA-seq was evaluated using an Agilent Technologies 2100 bioanalyzer.

Reverse transcription (RT) was performed using M-MLV reverse transcriptase (Promega) with the HSVd-specific primer HSV-105M (5'-GCTGGATTCTGAGAAGAGTT$3^{\prime}$, complementary to $\mathrm{HSVd}$ residues $105-86$ ), at $42^{\circ} \mathrm{C}$ for $1 \mathrm{~h}$. The genome of HSVd was amplified by high-fidelity $P f u$ DNA polymerase (Tiangen Biotech, Beijing) using the primer pair HSV-78P (5'-AACCCGGGGCAACTCTTCTC-3', homologous to HSVd residues 78-95) and HSV-83 M (5'AACCCGGGGCTCCTTTCTCA-3', complementary to HSVd residues 83-66) as reported previously (Sano et al., 2001). The PCR products were cloned into the pTOPO-Blunt Vector in the Zero Background pTOPO-Blunt Cloning Kit (Aidlab, Beijing) as indicated by the manufacturer's instructions. The recombinant plasmids were transformed into Escherichia coli $\mathrm{DH} 5 \alpha$ cells, and positive clones were randomly selected for sequencing.

\section{Northern-Blot Hybridization}

Equal amounts of total RNA (2 $\mu \mathrm{g})$ from each sample were subjected to agarose gel electrophoresis. The fractionated RNAs in the gel were transferred to Hybond $\mathrm{N}+$ nylon membranes using a vacuum transfer system (Bio-Rad) in 2 $\times$ SSC buffer. Northern-blot hybridization was performed at $68^{\circ} \mathrm{C}$ overnight with $\mathrm{HSVd}$-specific full length probes that were synthesized with a DIG RNA labeling kit (Roche) according to the manufacturer's instructions. Immunological detection was performed by incubating the membrane with the chemiluminescent substrate and subsequent exposure to X-ray film (Kodak).

\section{Library Construction and RNA-Seq Analysis}

Libraries for RNA-seq were constructed from $3 \mu \mathrm{g}$ samples of RNA using the NEBNext ${ }^{\circledR}$ Ultra $^{\text {TM }}$ RNA Library Prep Kit for Illumina ${ }^{\circledR}$ (NEB) according to the supplied instruction manual. Different index codes were added to distinguish the libraries prepared from each sample. In brief, mRNA was purified from total RNA using poly-T attached to magnetic beads and then fragmented using divalent cations at high temperature in NEBNext First Strand Synthesis Reaction Buffer $(5 x)$. First-strand cDNA was synthesized with M-MLV reverse transcriptase (RNase $\mathrm{H}-$ ) (Promega) using random hexamer primers; second-strand cDNA was synthesized using DNA polymerase I (Promega). The remaining overhangs of the double-stranded cDNA were converted into blunt ends using the exonuclease/polymerase activities of DNA polymerase (Promega). After adenylation of the $3^{\prime}$ termini, the cDNA fragments were ligated to NEBNext adaptors containing a hairpin loop structure. The resulting adaptor-ligated cDNA fragments (150-200 bp) were purified using the AMPure XP system (Beckman Coulter). The size-selected, adaptor-ligated cDNA was then incubated with $3 \mu \mathrm{L}$ of USER Enzyme (NEB) at $37^{\circ} \mathrm{C}$ for $15 \mathrm{~min}$ followed by $5 \mathrm{~min}$ at $95^{\circ} \mathrm{C}$. PCR amplification was performed using Phusion High-Fidelity DNA polymerase (NEB) with universal primers and an index $(\mathrm{X})$ primer. Finally, the PCR products purified using the AMPure XP system were run on the Agilent Bioanalyzer 2100 to assess library quality. DNA sequencing was performed by Novogene (Beijing) on an Illumina HiSeq 2500 sequencing instrument.

\section{Identification of Differentially Expressed Genes}

The DESeq R package (1.18.0) (Anders and Huber, 2010, 2013; Wang et al., 2010a) was used to compare the expression levels of host genes between the different samples. This software provides statistical routines for determining differential expression from digital gene expression (DGE) data using a model based on the negative binomial distribution; the resulting $P$-values were adjusted using the Benjamini and Hochberg approach (Benjamini and Hochberg, 1995) for controlling the false discovery rate (FDR). Those with an adjusted $P$-values $<0.05$ were considered as differentially-expressed genes.

\section{GO and KEGG Enrichment for Differentially Expressed Genes}

Gene Ontology (GO) enrichment analysis of differentiallyexpressed genes was carried out using the GOseq $\mathrm{R}$ package (Young et al., 2010), which corrects for gene length bias. GO terms with corrected $P$-values $<0.05$ were considered to be significantly enriched.

We applied the KEGG database (http://www.genome.jp/ kegg/) for understanding high-level functions and utilities of a biological system, such as the cell, the organism, and the ecosystem, from molecular-level information, especially largescale molecular datasets generated by genome sequencing and other high-throughput experimental technologies. We used the 
KOBAS software (Mao et al., 2005) to search for statistical enrichment of differentially-expressed genes in KEGG pathways. KEGG terms with corrected $P$-values $<0.05$ were considered to be significantly enriched in differentially-expressed genes.

\section{RT-qPCR}

RT-qPCR was used to test the accumulation of HSVd in HSVdinfected cucumber plants and to validate the expression levels of cucumber genes in those samples analyzed by RNA-seq. Equal amounts of total RNA $(2 \mu \mathrm{g})$ from each sample were used for cDNA synthesis by M-MLV reverse transcriptase (Promega) with random hexamer primers (Takara). The primers used for qPCR were designed using Integrated DNA Technologies (IDT) SciTools ${ }^{\circledR}$ Web Tools (PrimerQuest ${ }^{\circledR}$ program, IDT, Coralville, USA, http://www.idtdna.com/Scitools) except those for CsRDR1a, CsRDR1b, CsRDR1c1 (Leibman et al., 2017), CsPR1 (Kuzniak et al., 2015), and CsEF1 $\alpha$ (Wan et al., 2010) genes (Table S1). qPCR was performed on a MyGo Pro RealTime PCR System (IT-IS Life Science Ltd., UK) using GoTaq ${ }^{\circledR}$ qPCR Master Mix containing SYBR ${ }^{\circledR}$ Green I (Promega) as instructed. The cucumber EF1 $\alpha$ gene was used as an internal reference gene for normalization of expression levels (Wan et al., 2010).

\section{Photosynthetic Rate Measurements}

The photosynthetic rates of cucumber plants were measured with an LI-6400 Portable Photosynthesis System (LI-COR Biosciences, Inc., USA) as instructed by the manufacturer. Artificial illumination was supplied to the leaf from the system red-blue LED light source, and the photosynthetic rate was monitored as follows: six plants were randomly selected from each treatment and the temperature in the sample chamber was set to $(28 \pm 0.5)^{\circ} \mathrm{C}$. The level of photosynthetically active radiation (PAR) was then reduced in a step-wise fashion from 2,000 to $0 \mu \mathrm{mol} /\left(\mathrm{m}^{2} \cdot \mathrm{s}\right)$, and measurements were made once the leaf had attained a steady state. The resulting data were normalized using the total leaf area.

\section{RESULTS}

\section{HSVd Sequence Variants Induce Different Symptoms in Cucumber}

As shown in Figure 1A, there are four nucleotide differences between variants HSVd-h and HSVd-g54. When in vitro transcribed infectious transcripts corresponding to these two variants were inoculated into cucumber seedlings, they induced the appearance of either mild (HSVd-h) or severe (HSVd-g54) symptoms in the foliage (Figure 1B). Leaves of HSVd-h-infected plants were rough and mild wrinkled, whereas those infected with HSVd-g54 were severely wrinkled and distorted. Plants infected with HSVd-g54 also had shorter internodes than did those infected with HSVd-h (Figure 2A), resulting in reduced height of HSVd-g54-infected plants compared with those infected with HSVd-h. The timing of symptom expression was also different for these two variants. For example, all plants inoculated with HSVd-g54 displayed visible symptoms at $18 \mathrm{dpi}$, whereas those inoculated with HSVd-h displayed visible symptoms only at 22 dpi (Figure 2B). Similar results were observed in three independent experiments, confirming that HSVd-h and HSVd-g54 behave as mild and severe variants in cucumber, respectively.

The accumulation levels of HSVd-h and HSVd-g54 progeny in cucumber plants were compared by northern-blot hybridization and RT-qPCR. HSVd titers in HSVd-g54-infected plants were about 15- and 4-fold higher than those in HSVd-h-infected plants at 14 and 28 dpi (Figures 2C,D, Figure S1). To examine the stability of these two variants, HSVd cDNAs were amplified from two plants infected with each variant at 14 and 28 dpi. Five sequences were obtained from each sample, and comparison with the original sequences failed to reveal the presence of any mutants in HSVd-h- and HSVd-g54-infected plants.

\section{RNA-Seq Analysis of HSVd-g54- and HSVd-h-Infected Cucumber}

To compare the interaction of HSVd-h and HSVd-g54 with cucumber at the transcriptional level, non-inoculated cotyledon at $2 \mathrm{dpi}$ and top leaves at 14 and $28 \mathrm{dpi}$ of cucumber plants infected with the two HSVd variants were collected for transcriptome analysis. Next generation sequencing generated $34.33-48.28$ million raw reads per sample. After low quality reads and adapter sequences were removed, 33.29-46.67 million clean reads remained for each sample. Quality control parameters (Table S2) indicated that the resulting gene transcript data were reliable. In addition, the correlation of gene expression patterns and levels between biologically repeated samples was quite consistent, and $R^{2}$ values were between 0.956 (between biologically repeated HSVd-g54 infected plants at $28 \mathrm{dpi}$ ) and 0.994 (between biologically repeated HSVd-h infected plants at $2 \mathrm{dpi}$ ) (Figure S2), indicating high repeatability of the sequencing.

\section{Comparison of Gene Expression in Plants Infected with HSVd-g54 and HSVd-h}

Prior to identifying the differentially expressed genes (DEGs) associated with HSVd-g54 and HSVd-h infection in cucumber, gene expression levels in the different samples were calculated using FPKM (expected number of Fragments Per Kilobase of transcript sequence per Million base pairs sequenced) (Trapnell et al., 2010). The number of genes expressed in each sample was more than 19,000, which represents most of the genes predicted from sequence analysis of the cucumber genome (Huang et al., 2009). Expression levels of these genes in the different treatments were compared using the DEGSeq package (Anders and Huber, 2010).

HSVd-h and HSVd-g54 infection triggered different patterns of changes in gene expression in cucumber plants. HSVd-h induced very few DEGs at 2 and $14 \mathrm{dpi}$, but this number suddenly increased to 2599 at 28 dpi. HSVd-g54, in contrast, induced the highest number of DEGs (1428) at $2 \mathrm{dpi}$, and this number then gradually fell with time (Figure 3A, Table S3). Figure 3B compares these trends in the number of up- and down-regulated genes in cucumber plants infected with HSVd-h and HSVd-g54 in greater detail, and a heat cluster map (Figure 3C) shows 


\section{A}

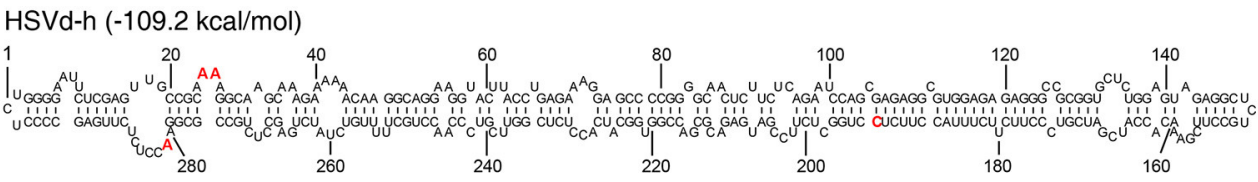

HSVd-g54 (-106.3 kcal/mol)

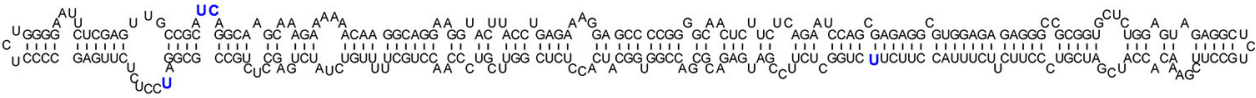

B
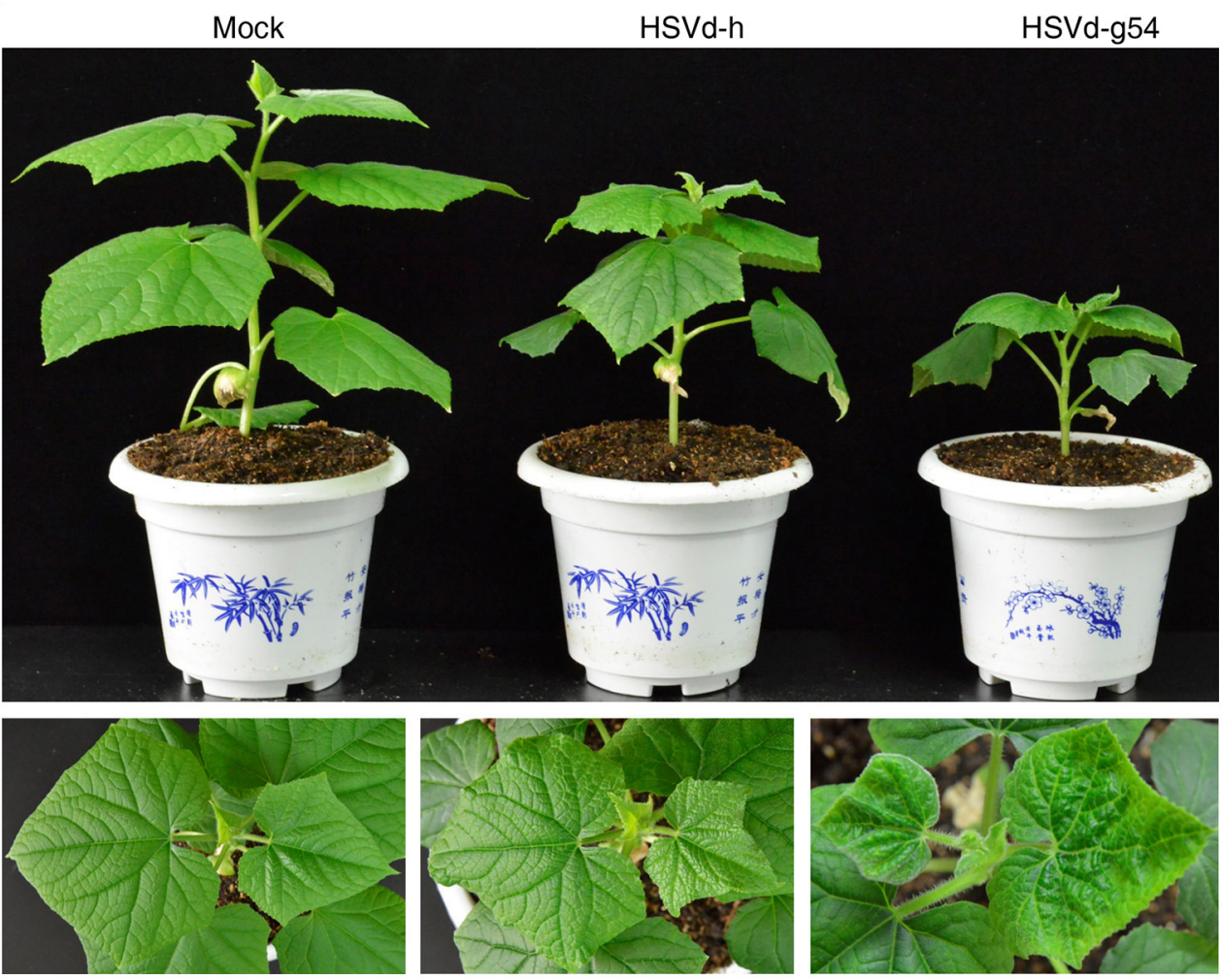

FIGURE 1 | Secondary structures of hop stunt viroid variants HSVd-h and HSVd-g54, and disease symptoms of cucumber plants infected with HSVd-h and HSVd-g54. (A) The positions of nucleotides in HSVd-g54 that differ from HSVd-h are shown in bold type. (B) Differences in plant height and leaf morphology among cucumber plants inoculated with sodium phosphate buffer (mock), HSVd-h, and HSVd-g54. Note that the leaves of plants infected with HSVd-g54 were more wrinkled than those infected with HSVd-h.

that the differences in DEGs expression patterns among three treatments at 14 and $28 \mathrm{dpi}$ were larger than those at $2 \mathrm{dpi}$.

To validate the RNA-seq results, ten genes showing different levels of expression (Table S4) were selected, and their expression levels were analyzed by RT-qPCR using gene-specific primers (Table S1). Results showed that the expression profiles of eight of these ten genes were very similar to those determined by RNA-seq (Table S4, Figure 4).

\section{Gene Ontology Enrichment Analysis}

To analyze the function of DEGs showing up- or downregulation in response to HSVd-h or HSVd-g54 infection, we performed GO enrichment analysis. Individual GO terms were considered to be enriched if their corrected $P$-value was $<0.05$, and Table S5 contains a list of all enriched GO terms.
Table 1 shows the top 20 enriched GO terms (all GO terms are listed if the number of enriched GO terms was $<20$ ) for the up-regulated DEGs at various time points. Note that the up-regulated DEGs are mainly enriched in protein phosphorylation, protein modification processes, protein kinase activity, phosphotransferase activity, and ribonucleotide binding in cucumber plants infected with the mild variant HSVd-h at 14 and 28 dpi. Plants infected with the severe variant HSVd-g54 showed a very similar pattern of GO terms enrichment at 2 and $14 \mathrm{dpi}$, while the four enriched GO terms at 28 dpi were very different (Table 1).

Table 2 contains a comparable list of enriched GO terms for the down-regulated DEGs. While no GO terms were enriched for down-regulated DEGs in HSVd-h-infected plants at 2 and $14 \mathrm{dpi}, 20 \mathrm{GO}$ terms related to microtubule-based movement, 

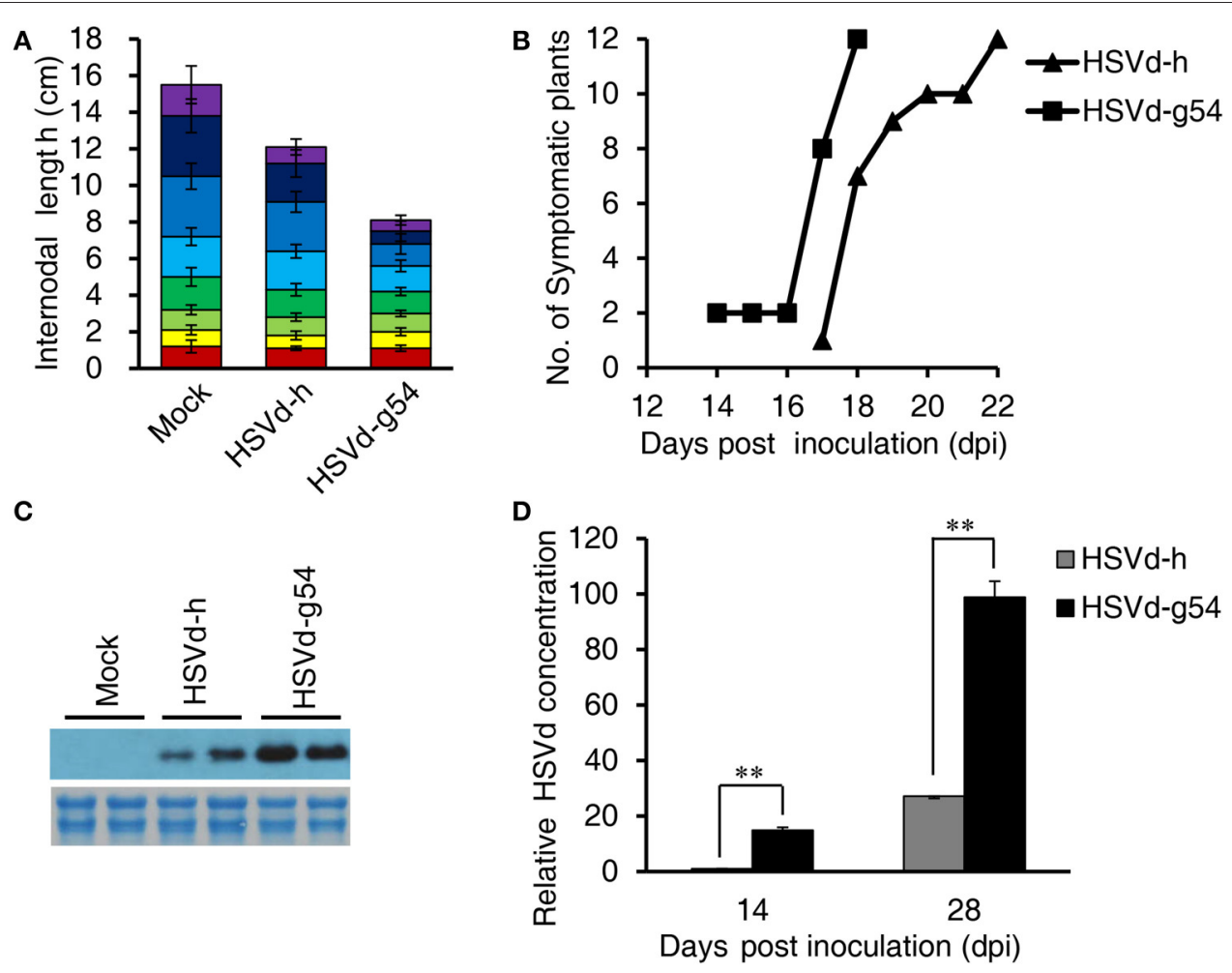

FIGURE 2 | Effect of HSVd-h and HSVd-g54 infection on cucumber growth and development. (A) Internode lengths of cucumber plants at 28 dpi. Individual colored bars represent the lengths of successive internodes, with the first internode shown at the bottom. Measurements were taken from 12 cucumber plants per treatment, and data are expressed as the mean \pm standard deviation. Error bars indicate the standard deviations of 12 replicates. (B) Time course of symptom appearance in cucumber plants following viroid inoculation. (C) Comparison of the concentrations of the two HSVd variants at 28 dpi by Northern blotting. Total RNAs (2 $\mu \mathrm{g})$ were fractionated by electrophoresis in 1.5\% (w/v) agarose gels and used for loading controls (below the hybridization signals). (D) Relative levels of viroid RNA as determined by RT-qPCR. Experiments were performed in triplicate and the data are expressed as the mean \pm standard deviation. Error bars indicate the standard deviations of three biological replicates of RT-qPCR analysis. Double asterisks indicate statistically significant differences compared with the control at $p<0.01$ in Student's $t$-test.

microtubule binding, and microtubule motor activity were enriched at $28 \mathrm{dpi}$. Conversely, no GO terms were enriched for down-regulated DEGs in HSVd-g54-infected plants at 2 dpi, but $11 \mathrm{GO}$ terms related to photosystem, thylakoid, photosynthetic membrane, and the photosystem I reaction center were enriched at 14 dpi.

\section{KEGG Enrichment Analysis}

Many different gene-encoded products function in vivo as part of one or more biochemical pathways. To determine in which major metabolic and signal transduction pathways might be disrupted by HSVd infection, we performed a KEGG enrichment analysis (Table 3, Table S6).

Four KEGG pathways were enriched in up-regulated DEGs: the pentose phosphate pathway, plant hormone signal transduction, plant-pathogen interaction, and endocytosis. Plant hormone signal transduction and plant-pathogen interaction were the main pathways affected, because they were enriched in plants infected with HSVd-g54 at 2 and $28 \mathrm{dpi}$ as well as HSVd-h at $28 \mathrm{dpi}$. Hormone signal transduction was enriched in plants infected with HSVd-g54 at $14 \mathrm{dpi}$, while the pentose phosphate pathway and endocytosis were enriched at 2 dpi in plants infected with HSVd-h and HSVd-g54 (Table 3).

Thirteen KEGG pathways were enriched in down-regulated DEGs. Photosynthesis-antenna proteins were enriched in plants infected with HSVd-h at 2 dpi and HSVd-g54 at 2 and 14 dpi. Other down-regulated pathways were enriched only at a single time point (Table 3).

\section{HSVd Infection Induces Expression of Many Basal Defense Response-Related Genes}

Plants have evolved a variety of defense responses to prevent or limit disease. Many of these responses are activated locally at the site of infection and then spread systemically when a plant is under pathogen attack (Whitham et al., 2006; Pallas and García, 2011; Allie et al., 2014). This initial response is usually termed basal or broad spectrum immunity and may be sufficient to combat a viral pathogen.

Activation of the defense response results from several possible signaling pathways, including reactive oxygen species (ROS), signaling molecules, and pathogenesis-related proteins 


\section{A}

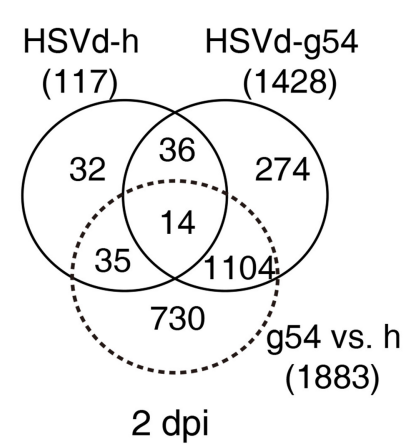

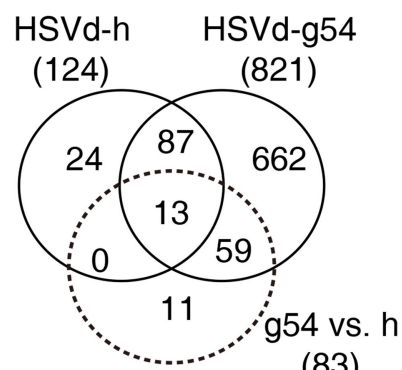

(83)

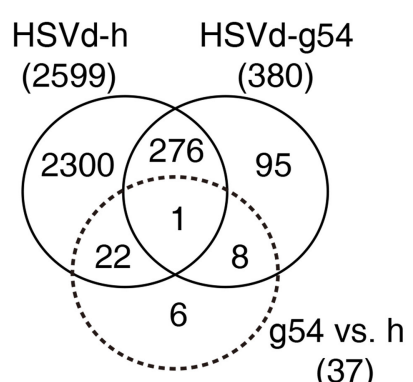

(37)

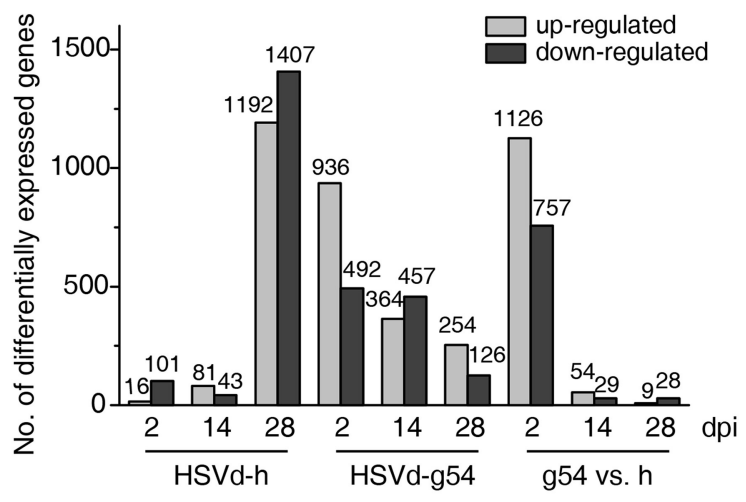

C

$28 \mathrm{dpi}$

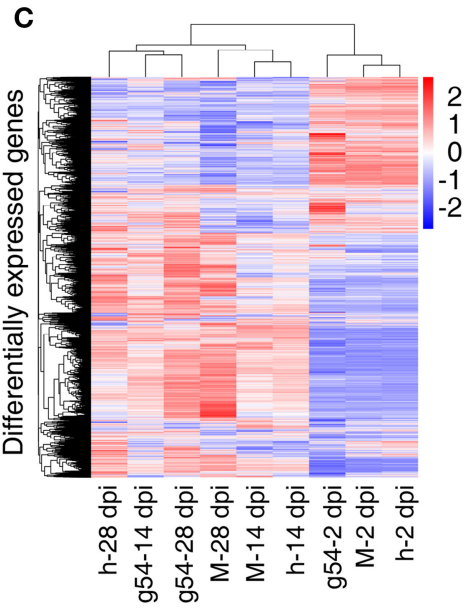

FIGURE 3 | Comparison of differentially expressed genes (DEGs) in response to HSVd-g54 and HSVd-h infection of cucumber. (A) Venn diagrams showing unique and common DEGs in different comparisons at 2, 14, and 28 dpi. (B) The number of up- and down-regulated DEGs in different comparisons. (C) A heat cluster map of cucumber DEGs resulting from HSVd infection. Red, up-regulated genes; blue, down-regulated genes; the red and blue colors represent the values of log 10 (FPKM + 1) from high to low. M, mock infected plants; h, HSVd-h; g54, HSVd-g54.

(PR proteins), leading to biochemical and morphological alterations such as cell wall reinforcement and transmembrane reconfiguration in the host (Fagard et al., 2007; Blomster et al., 2011). KEGG pathway analysis revealed that many genes involved in plant basal defense responses were expressed at higher levels in HSVd-inoculated cucumber plants (Table S7). Compared with mock-inoculated plants, genes encoding cyclic nucleotide gated channels (CNGCs), calcium-dependent protein kinase (CDPK), respiratory burst oxidase (Rboh), calciumbinding protein CML (CaMCML), WRKY transcription factor 33 (WRKY33), LRR receptor-like serine/threonine-protein kinase FLS2 (FLS2), mitogen-activated protein kinase kinase kinase 1 (MEKK1), brassinosteroid insensitive 1-associated receptor kinase 1 (BAK1/BKK1), the basic form of PR1, and mitogenactivated protein kinase kinase 4/5 (MKK4/5) were all upregulated at different time points (Table S7). The number of upregulated DEGs involved in basal defense responses increased over the time course of the experiment for the HSVd-h vs. mock control, while the number of basal defense response-related genes declined in the HSVd-g54 vs. mock control. It should be noted that the PR1 gene was expressed at extremely low levels in mock-inoculated cucumber plants but was up-regulated in plants infected with either HSVd-h or HSVd-g54 at 14 and 28 dpi (Table S7).

\section{HSVd Infection Represses Expression of Photosynthesis-Related Genes}

To better understand the molecular effects of HSVd infection in cucumber, we annotated the DEGs identified in HSVd-hand HSVd-g54-inoculated tissue harvested at all time points using the GO and KEGG terms. For HSVd-g54-infected systemic leaves harvested at $14 \mathrm{dpi}$, almost all of the down-regulated GO terms were in the cellular component category. These genes were involved in chloroplast functions including photosystem I and II (PS I, PS II), thylakoids and photosynthesis (Table 2). At $2 \mathrm{dpi}$ the most strongly down-regulated KEGG terms in the HSVd-h vs. mock and HSVd-g54 vs. mock controls were photosynthesis-antenna proteins (Table 3). At 14 dpi two of the down-regulated KEGG terms in the HSVd-g54 vs. mock control were photosynthesis and photosynthesis-antenna proteins. At 2 dpi, only the genes encoding the light-harvesting chlorophyll (LHC) complex proteins Lhca2, Lhca4, Lhcb1, Lhcb2, and Lhcb3 were down-regulated in HSVd-h-infected cucumber plants; in the HSVd-g54-infected plants, in contrast, genes encoding Lhca1, 


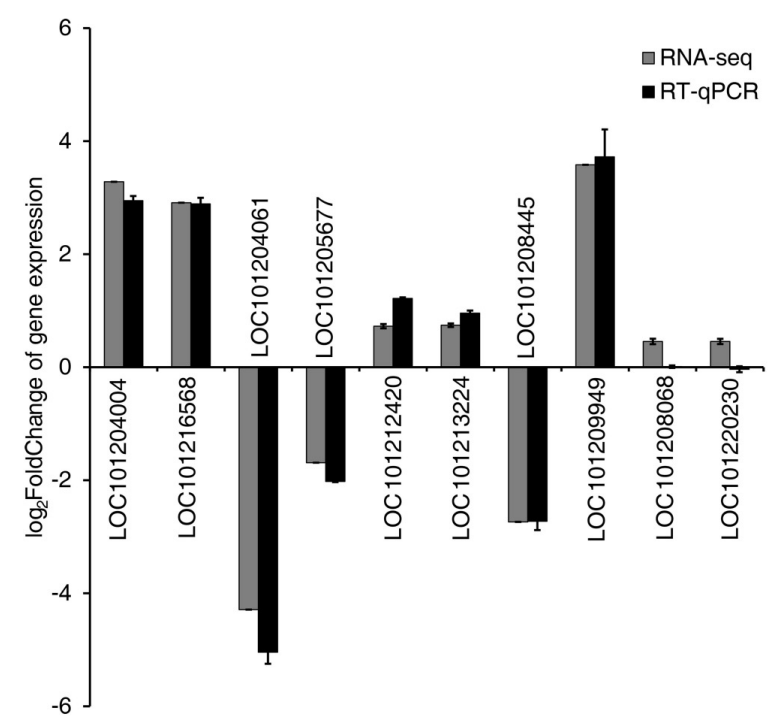

FIGURE 4 | Validation of RNA-seq results by quantitative real-time PCR (RT-qPCR). Expression patterns of 10 representative genes as determined by RT-qPCR and RNA-seq. Normalization for RT-qPCR was performed using expression of the EF1 $\alpha$ gene as an internal reference. Error bars on the black boxes indicate the standard deviations of three biological replicates of RT-qPCR analysis. The bars on the gray boxes indicate the false discovery rates from the two biological replicates of RNA-seq analysis.

Lhca2, Lhca3, Lhca4, Lhcb1, Lhcb2, Lhcb3, Lhcb5, and Lhcb6 were down-regulated. At $14 \mathrm{dpi}$, genes encoding photosystem I and II proteins PsbO, PsbP, PsbQ, PsbW, PsbY, PsaD, PsaE, PsaF, PsaG, PsaH, PsaK, PsaL, PsaN, and PsaO, as well as genes encoding photosynthetic electron transport proteins PetE and PetH and the F-type ATPase subunits $b$ and $\gamma$ were downregulated in HSVd-g54-infected plants.

To determine whether or not these changes in expression levels affected photosynthesis, the photosynthetic rates of cucumber plants inoculated with HSVd-h and HSVd-g54 were measured with a LI-6400 Portable Photosynthesis System at 14 and $28 \mathrm{dpi}$ and then compared to those of mock-inoculated plants normalized according to the total leaf area. The results showed that the photosynthetic rate in HSVd-h- and HSVd-g54infected plants declined dramatically as compared to the control plants at $14 \mathrm{dpi}$. Interestingly, differences between plants infected with HSVd-h and HSVd-g54 were not statistically significant (Figure 5). At $28 \mathrm{dpi}$, photosynthetic rates in cucumber plants infected with HSVd-g54 decreased sharply compared to those in HSVd-h-infected or mock-inoculated plants: no significant difference was observed between the HSVd-h and mockinoculated plants. These results indicated that infection with HSVd inhibits photosynthesis in cucumber plants.

\section{HSVd Infection Induces Expression of Salicylic Acid-Related Genes}

Phytohormones play critical roles in modulating plant growth and development, and infection by many viruses and viroids can affect several kinds of phytohormone signaling pathways as well as their biosynthesis and catabolism, resulting in the appearance of disease symptoms in the infected plants (Whenham et al., 1986; Owens et al., 2012). In this study, we also used KEGG enrichment analysis to identify DEGs related to phytohormone signal transduction. In cucumber plants infected with HSVd-h, phytohormone signal transduction was the top KEGG term at both 14 and 28 dpi. Four DEGs were involved in the IAA, ET, $\mathrm{JA}$, and SA signal transduction pathways at $14 \mathrm{dpi}$; at $28 \mathrm{dpi}$ the number of DEGs increased to 57, including most of the major phytohormone signaling pathways (Table S8). Compared with mock-inoculated plants, cucumber plants infected with HSVd-g54 had 35, 18, and 13 DEGs related to phytohormone signal transduction at 2, 14, and $28 \mathrm{dpi}$, respectively (Table S8). Especially noteworthy was the up-regulation of genes encoding the main components of the SA signaling pathway in HSVdinfected plants. NPR1 (Non-expressor of pathogenesis-related genes 1) was up-regulated in HSVd-h-infected plants at $28 \mathrm{dpi}$ and HSVd-g54 infected plants at $14 \mathrm{dpi}$. PR1, which is also known as a marker gene for SA (Divi et al., 2010; Ford et al., 2010), was up-regulated in both HSVd-h- and HSVd-g54-infected plants at 14 and 28 dpi (Figure 6, Table S3). This suggested that HSVdinfected plants may contain higher concentrations of SA than healthy cucumber plants.

\section{HSVd Infection Induces Expression of CsRDR1 Genes}

RNA silencing not only plays a critical role in plant antiviral response but is also directly involved in viral disease induction in plants (Navarro et al., 2012; Wang et al., 2012). We therefore screened the cucumber DEGs associated with HSVd infection for the presence of RNA silencing-related genes encoding DicerLike enzymes (DCL), Argonaute proteins (AGO), and RDR. DCL1 and DCL4 were slightly up-regulated, and AGO7, AGO10, and AGO16 were down-regulated more than two-fold at 28 dpi in HSVd-h-infected plants. Notably, the expression of genes encoding components of RDR 1 was changed dramatically in both HSVd-h- and HSVd-g54-infected plants at various time points (Table 4). Cucumber RDR1 consists of CsRDR1a, CsRDR1b, CsRDR1c1, and CsRDR1c2 (Leibman et al., 2017). CsRDR1a expression was slightly stimulated in HSVd-h-infected cucumber plants at $28 \mathrm{dpi}$, and expression of CsRDR $1 b$ increased slightly in HSVd-h-infected plants at $28 \mathrm{dpi}$ and HSVd-g54infected plants at 14 and $28 \mathrm{dpi}$ (Table 4). In contrast to $C s R D R 1 a$ and $C s R D R 1 b$, expression of $C s R D R 1 c 1$ and $C s R D R 1 c 2$ increased dramatically in HSVd-infected cucumber plants. At $28 \mathrm{dpi}$, expression of CsRDR1c1 was highly up-regulated in both HSVd-h- and HSVd-g54-infected plants; expression of CsRDR1c2 was significantly induced in both HSVd-h- and HSVd-g54-infected cucumber plants at both 14 and $28 \mathrm{dpi}$ (Table 4). These results obtained using RNA-seq were validated by RT-qPCR, and data summarized in Table 4 show that both methods detected similar changes in CsRDR1 gene expression.

\section{DISCUSSION}

In this study we examined the changes in the cucumber transcriptome that occur following infection by mild (HSVd-h) 
TABLE 1 | The top 20 (>20) or all ( $\leq 20)$ enriched GO terms for the up-regulated DEGs in HSVd-infected cucumber plants.

\begin{tabular}{|c|c|c|c|c|c|c|c|}
\hline \multirow[t]{3}{*}{ GO accession } & \multirow[t]{3}{*}{ Description } & \multirow[t]{3}{*}{ Term type* } & \multicolumn{5}{|c|}{ 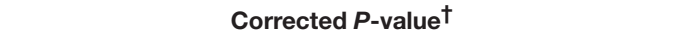 } \\
\hline & & & \multicolumn{2}{|c|}{ HSVd-h } & \multicolumn{3}{|c|}{ HSVd-g54 } \\
\hline & & & 14 dpi & 28 dpi & 2 dpi & 14 dpi & 28 dpi \\
\hline GO:0006468 & Protein phosphorylation & $\mathrm{BP}$ & 3.76E-04 & $1.84 \mathrm{E}-11$ & $7.78 \mathrm{E}-15$ & $4.15 \mathrm{E}-12$ & \\
\hline GO:0016310 & Phosphorylation & $\mathrm{BP}$ & 6.95E-04 & 1.07E-09 & $6.41 \mathrm{E}-13$ & $4.24 \mathrm{E}-11$ & \\
\hline GO:0006464 & Cellular protein modification process & $\mathrm{BP}$ & 2.53E-03 & 3.71E-08 & $2.85 \mathrm{E}-14$ & $1.01 \mathrm{E}-10$ & \\
\hline GO:0036211 & Protein modification process & $\mathrm{BP}$ & 2.53E-03 & 3.71E-08 & $2.85 \mathrm{E}-14$ & $1.01 \mathrm{E}-10$ & \\
\hline GO:0043412 & Macromolecule modification & $\mathrm{BP}$ & $1.21 \mathrm{E}-02$ & 6.63E-06 & $1.97 \mathrm{E}-11$ & 1.15E-09 & \\
\hline GO:0006796 & Phosphate-containing compound metabolic process & $\mathrm{BP}$ & 3.91E-02 & 3.35E-07 & $6.04 \mathrm{E}-11$ & 8.66E-09 & \\
\hline GO:0006793 & Phosphorus metabolic process & $\mathrm{BP}$ & 3.91E-02 & 3.35E-07 & $6.04 \mathrm{E}-11$ & 8.66E-09 & \\
\hline GO:0044267 & Cellular protein metabolic process & $\mathrm{BP}$ & & & 8.55E-06 & 7.89E-06 & \\
\hline GO:0007154 & Cell communication & $\mathrm{BP}$ & & 7.66E-05 & & & \\
\hline GO:0050794 & Regulation of cellular process & $\mathrm{BP}$ & & & & & 3.93E-02 \\
\hline GO:0050789 & Regulation of biological process & $\mathrm{BP}$ & & & & & 3.93E-02 \\
\hline GO:0005578 & Proteinaceous extracellular matrix & $\mathrm{CC}$ & & & $1.31 \mathrm{E}-08$ & & \\
\hline GO:0031012 & Extracellular matrix & $\mathrm{CC}$ & & & 7.76E-08 & & \\
\hline GO:0004672 & Protein kinase activity & MF & $6.78 \mathrm{E}-05$ & $4.12 \mathrm{E}-12$ & $7.78 \mathrm{E}-15$ & $1.25 \mathrm{E}-12$ & \\
\hline GO:0016773 & Phosphotransferase activity, alcohol group as acceptor & MF & 3.76E-04 & 1.98E-09 & $1.79 \mathrm{E}-13$ & $3.26 \mathrm{E}-11$ & \\
\hline GO:0016301 & Kinase activity & MF & $6.95 E-04$ & 9.20E-09 & $1.24 \mathrm{E}-12$ & $8.76 \mathrm{E}-10$ & \\
\hline GO:0016772 & Transferase activity, transferring phosphorus-containing groups & MF & $9.78 \mathrm{E}-03$ & 2.05E-05 & $6.18 \mathrm{E}-10$ & $3.75 \mathrm{E}-08$ & \\
\hline GO:0016740 & Transferase activity & MF & & & 7.45E-08 & & \\
\hline GO:0097367 & Carbohydrate derivative binding & MF & & & 8.55E-06 & $1.50 \mathrm{E}-05$ & \\
\hline GO:0032553 & Ribonucleotide binding & MF & & & 1.35E-05 & & \\
\hline GO:0035639 & Purine ribonucleoside triphosphate binding & MF & 4.47E-02 & & & & \\
\hline GO:0005524 & ATP binding & MF & 4.47E-02 & & 1.67E-05 & 7.63E-06 & \\
\hline GO:0001883 & Purine nucleoside binding & MF & 4.47E-02 & 8.32E-05 & & 1.63E-05 & \\
\hline GO:0032549 & Ribonucleoside binding & MF & 4.47E-02 & 8.32E-05 & & 1.63E-05 & \\
\hline GO:0032550 & Purine ribonucleoside binding & MF & 4.47E-02 & 8.32E-05 & & 1.63E-05 & \\
\hline GO:0001882 & Nucleoside binding & MF & 4.47E-02 & 8.32E-05 & & & \\
\hline GO:0032555 & Purine ribonucleotide binding & MF & 4.47E-02 & 8.32E-05 & & & \\
\hline GO:0032559 & Adenyl ribonucleotide binding & MF & 4.47E-02 & $4.72 \mathrm{E}-05$ & 8.86E-06 & 6.07E-06 & \\
\hline GO:0030554 & Adenyl nucleotide binding & MF & & $6.26 \mathrm{E}-05$ & 1.08E-05 & 4.95E-06 & \\
\hline GO:0017076 & Purine nucleotide binding & MF & $4.62 \mathrm{E}-02$ & $1.94 \mathrm{E}-04$ & & 1.63E-05 & \\
\hline GO:0001071 & Nucleic acid binding transcription factor activity & MF & & & & & 1.60E-02 \\
\hline GO:0003700 & Transcription factor activity, sequence-specific DNA binding & MF & & & & & 1.60E-02 \\
\hline
\end{tabular}

${ }^{\star} B P$, biological process; CC, cellular component; MF, molecular function.

${ }^{\dagger}$ No GO term in the up-regulated DEGs was enriched in HSVd-h-infected cucumber plants at 2 dpi.

and severe (HSVd-g54) variants of HSVd. An earlier study compared differences in the tomato transcriptome caused by infection with mild and severe strains of PSTVd using subtracted cDNA libraries (Itaya et al., 2002). Two other studies focused on the changes in host gene expression following either infection by citrus exocortis viroid (CEVd) (Rizza et al., 2012) or PSTVd infection in two different tomato cultivars (Owens et al., 2012). Each of these studies used macroarray or microarray analysis to assess the host response to a single viroid variant at a single time point. Here, we have used RNA-seq analysis to compare changes in cucumber gene expression associated with infection by both a mild and severe variants of HSVd during the early (2 dpi), middle (pre-symptomatic, $14 \mathrm{dpi}$ ), and late (post-symptomatic, $28 \mathrm{dpi}$ ) stages of infection. The results described highlight the transcriptomic changes caused by HSVd infection, changes affecting genes involved in basal defense responses, photosynthesis, and SA regulation as well as in CsRDR1.

\section{Viroid Infection Triggers Basal Defense Responses}

Plants have evolved an innate immune system that recognizes invading microbes and initiates an effective defense response against pathogen attack (Wu et al., 2014). These are known as basal defense responses. When pathogen invasion occurs, plants translate perception of the pathogen into signal cascades involving CNGCs that raise $\mathrm{Ca}^{2+}$ concentrations in the cytosol and activate $\mathrm{CaMs}$ and/or CaM-like proteins (CMLs) (Ali et al., 2007). CDPKs are another major type of $\mathrm{Ca}^{2+}$ 
TABLE 2 | All enriched GO terms for the down-regulated DEGs in HSVd-infected cucumber plants.

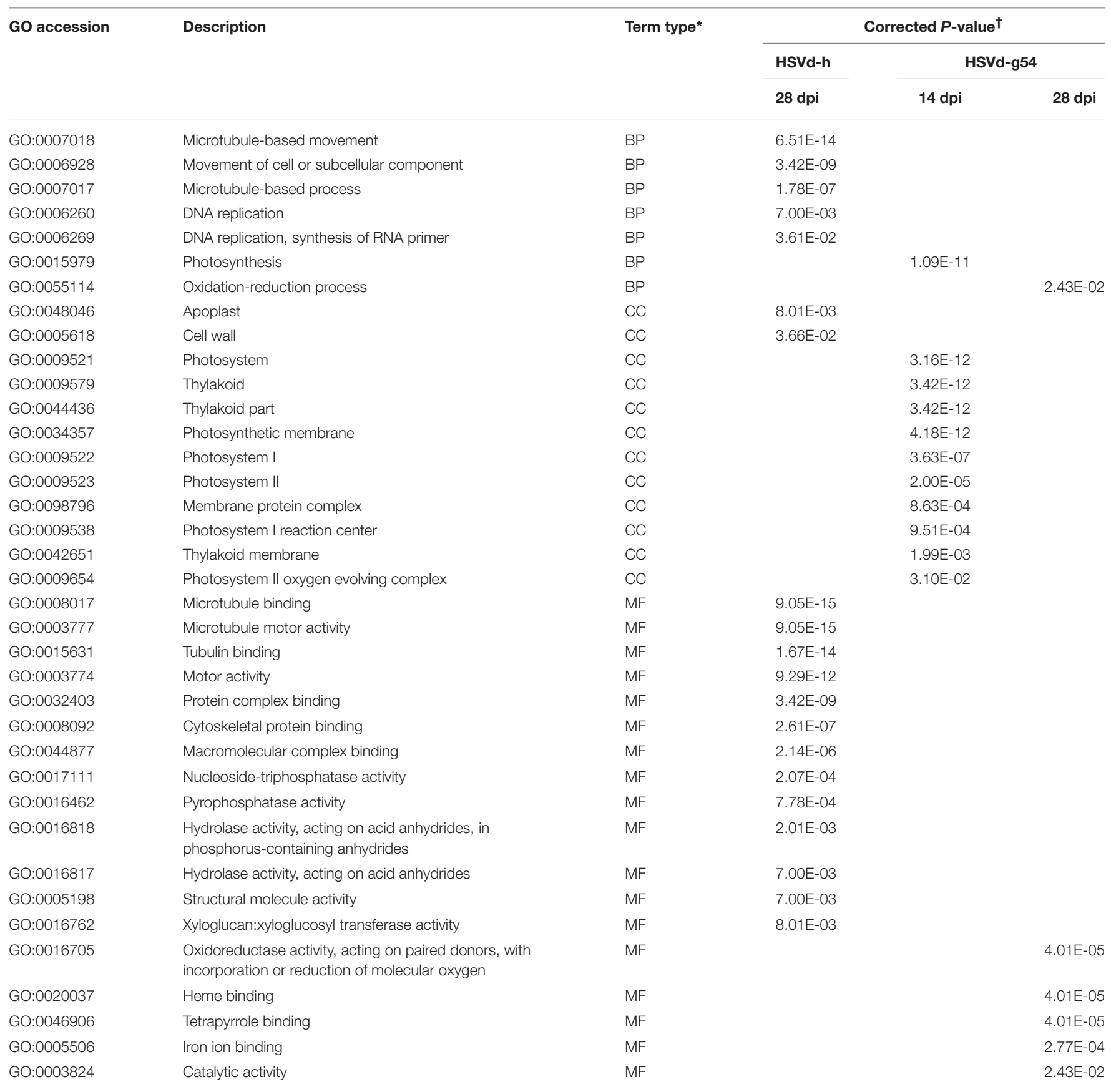

${ }^{*} B P$, biological process; CC, cellular component; MF, molecular function.

${ }^{\dagger}$ No GO term in the down-regulated DEGs was enriched in HSVd-h-infected cucumber plants at 2 and 14 dpi and HSVd-g54 at 2 dpi.

sensor in plants, and they can be activated by $\mathrm{Ca}^{2+}$. CDPK cascades are initiated downstream of the activated receptor complex and further mediate defense gene expression (Wu et al., 2014).

Arabidopsis CDPK1, 2, 4, and 11 strongly phosphorylate both RbohD and RbohF in vitro and mediate AvrRpm1-induced ROS production (Gao et al., 2013). Expression of CNGCs, CaMs/CMLs, CDPKs, and Rboh was changed in cucumber plants infected with HSVd-h at $28 \mathrm{dpi}$ and HSVd-g54 at 2 and 14 dpi (Table S7). Expression of FLS2, an LRR receptor-like kinase usually involved in perception of the bacterial elicitor flagellin, was also activated by HSVd infection, and it can associate with BAK1 to form the FLS2/BAK1 complex (GómezGómez and Boller, 2000; Wu et al., 2014). The signal can then be passed from FLS2 to MEKK1, and accumulating evidence suggests that in Arabidopsis perception activates two branches 
TABLE 3 | KEGG pathway enrichment of DEGs from HSVd-infected cucumber plants.

\begin{tabular}{|c|c|c|c|c|c|c|c|c|c|c|c|c|c|}
\hline \multirow[t]{3}{*}{ Pathway } & \multirow[t]{3}{*}{ ID } & \multicolumn{6}{|c|}{ HSVd-h } & \multicolumn{6}{|c|}{ HSVd-g54 } \\
\hline & & \multicolumn{2}{|c|}{2 dpi } & \multicolumn{2}{|c|}{14 dpi } & \multicolumn{2}{|c|}{$28 \mathrm{dpi}$} & \multicolumn{2}{|r|}{2 dpi } & \multicolumn{2}{|c|}{14 dpi } & \multicolumn{2}{|c|}{28 dpi } \\
\hline & & No.* & $q^{\dagger}$ & No. & $q$ & No. & $q$ & No. & $q$ & No. & $q$ & No. & $q$ \\
\hline \multicolumn{14}{|c|}{ UP-REGULATED ENRICHMENT KEGG PATHWAYS (CORRECTED P-VALUE < 0.05) } \\
\hline Pentose phosphate pathway & csv00030 & 2 & 3.03E-02 & & & & & & & & & & \\
\hline Plant hormone signal transduction & csv04075 & & & & & 44 & 1.03E-13 & 20 & 3.09E-02 & 13 & 2.74E-03 & 13 & 1.81E-06 \\
\hline Plant-pathogen interaction & csv04626 & & & & & 21 & 3.07E-06 & 28 & $1.47 \mathrm{E}-11$ & & & 5 & 3.87E-02 \\
\hline Endocytosis & csv04144 & & & & & & & 12 & 2.08E-02 & & & & \\
\hline \multicolumn{14}{|c|}{ DOWN-REGULATED ENRICHMENT KEGG PATHWAYS (CORRECTED $P$-VALUE < 0.05) } \\
\hline Photosynthesis - antenna proteins & csv00196 & 8 & 4.99E-09 & & & & & 14 & $8.00 \mathrm{E}-15$ & 10 & 1.46E-09 & & \\
\hline Glutathione metabolism & csv00480 & 7 & 1.44E-04 & & & & & & & & & & \\
\hline Protein processing in endoplasmic reticulum & csv04141 & 9 & 8.58E-04 & & & & & & & & & & \\
\hline Plant-pathogen interaction & csv04626 & & & 2 & $1.40 \mathrm{E}-02$ & & & & & & & & \\
\hline DNA replication & csv03030 & & & & & 16 & 3.47E-04 & & & & & & \\
\hline Porphyrin and chlorophyll metabolism & csv00860 & & & & & 11 & 1.96E-02 & & & & & & \\
\hline Ribosome & csv03010 & & & & & 42 & 4.05E-02 & & & & & & \\
\hline Plant hormone signal transduction & csv04075 & & & & & & & 15 & 2.27E-03 & & & & \\
\hline Photosynthesis & csv00195 & & & & & & & & & 21 & $1.85 \mathrm{E}-16$ & & \\
\hline Flavonoid biosynthesis & csv00941 & & & & & & & & & & & 4 & 3.98E-04 \\
\hline Biosynthesis of secondary metabolites & csv01110 & & & & & & & & & & & 17 & 7.16E-03 \\
\hline Phenylpropanoid biosynthesis & csv00940 & & & & & & & & & & & 6 & 1.35E-02 \\
\hline Phenylalanine metabolism & csv00360 & & & & & & & & & & & 5 & 1.86E-02 \\
\hline
\end{tabular}

${ }^{\star}$ No., Input number; ${ }^{\dagger} q$, Corrected P-value $(<0.05)$.

of the MAPK cascade: MEKK1/MEKKs-MKK4/5-MPK3/6 and MEKK1-MKK1/2-MPK4 (Tena et al., 2011; Wu et al., 2014).

Pathogen infection often leads to transcriptional changes in the plant host. A very mild response usually does not result in large-scale changes in gene expression patterns. However, a hypersensitive response (HR) is accompanied by massive increases in the levels of a large number of different proteins. Among these induced proteins are members of a group of PR proteins (Linthorst and Van Loon, 1991). When infected by apple stem grooving virus (ASGV), apple genes encoding PR proteins PR1 and PR1a were up-regulated (Chen et al., 2014); similarly, PR1 expression in tomato was also induced by infection with tomato spotted wilt virus (TSWV) (López-Gresa et al., 2016) and CEVd (Conejero et al., 1990; López-Gresa et al., 2016). The upregulation of cucumber $P R 1$ gene expression, which we observed following either HSVd-h or HSVd-g54 infection (Table S7), is consistent with the results of these previous studies.

\section{Viroid Infection Represses Plant Photosynthesis}

The photosynthetic rate in HSVd-infected cucumber plants was reduced (Figure 5). Many genes associated with photosynthesis were found to be down-regulated in cucumber plants infected with HSVd-h or HSVd-g54 compared to mock-inoculated plants. The organic compounds and energy required for plant growth comes from photosynthesis, and limiting photosynthesis may repress growth and development, which may be one of the causes of dwarfing observed in cucumber plants inoculated with HSVd, especially those inoculated with the severe mutant HSVd-g54.

Viroid infection could modify the structure or synthesis of chloroplast, which affect the efficiency of photosynthesis. There are many studies showing that virus infection represses photosynthesis in the host (Li et al., 2016). Virus infection can impair photosynthesis by decreasing carboxylation and mesophyll conductance (Sampol et al., 2003), reducing carboxylative efficiency (Pérez-Clemente et al., 2015), modifying the structure of chloroplasts, or reducing the number of active reaction centers (Prod'homme et al., 2001; Zechmann et al., 2003; Bhattacharyya et al., 2015; Otulak et al., 2015). Previous studies also reported that the chloroplast structure of viroid-infected plant tissues was modified, particularly thylakoid membrane abnormalities and paucity of grana in many viroid-host combinations (Hari, 1980; da Graça and Martin, 1981; Kojima et al., 1983; Momma and Takahashi, 1983). The infection of peach latent mosaic viroid (PLMVd) with a specific structural motif (12- to 13-nucleotide insertion) inhibited chloroplast development (Rodio et al., 2007). Microarray analysis indicated that viroid infection triggered changes in chloroplast, and its biogenesis-related genes were down-regulated (Owens et al., 2012; Rizza et al., 2012). In this study, we annotated the down-regulated genes associated with photosynthesis GO and KEGG terms. Proteins encoded by these genes included photosynthesis-antenna proteins (components of LHC in PS I and PS II), photosynthetic electron transport proteins PetE and $\mathrm{PetH}$, and the F-type ATPase subunits $\mathrm{b}$ and $\gamma$. Thus, 


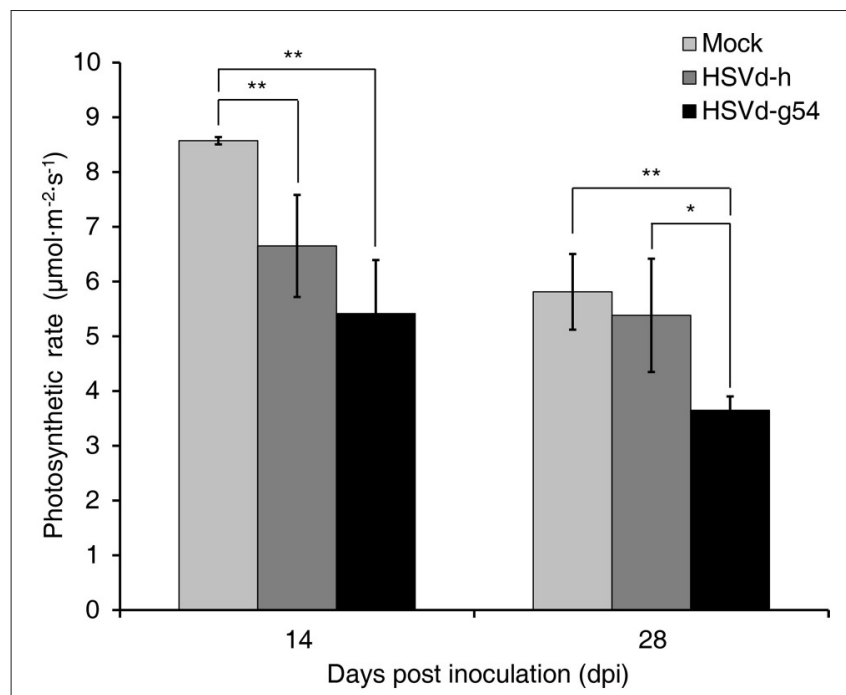

FIGURE 5 | Effect of HSVd infection on the rate of photosynthesis in cucumber. Experiments were performed on six cucumber plants, and the data are expressed as the mean \pm standard deviation. Error bars indicate the standard deviations of six replicates. Single or double asterisks indicate statistically significant differences compared with the control at $p<0.05$ or 0.01 , respectively, in Student's $t$-test.

HSVd infection down-regulates the expression of several genes involved in photosynthesis, genes that could explain the decrease in photosynthetic rate observed in the infected plants.

\section{Role of SA in the Interaction between Viroid and Plants}

HSVd infection induces the expression of the SA marker gene PR1 in cucumber plants, and SA may be involved in the interaction between viroid and plants. During infection by viruses and viroids, disruption of normal host developmental physiology is often associated with alterations in phytohormone production, accumulation, and signaling (Whenham et al., 1986; Zhu et al., 2005; Hammond and Zhao, 2009; Owens et al., 2012; Rodriguez et al., 2014; Collum and Culver, 2016).

In our study, most of the major plant hormone signal transduction pathways were found to be affected at different times after infection by the two HSVd variants tested. And this indicated HSVd infection changed many plant hormone signal transduction pathways simultaneously, and the relationship between viroid, phytohormone, and host plant is complex. However, some regulation by plant hormones was still present. For example, genes encoding some components of the SA signal transduction pathway were up-regulated by both HSVd-h and HSVd-g54 at 14 and $28 \mathrm{dpi}$; this was especially true for PR1, a known SA marker gene (Divi et al., 2010; Ford et al., 2010), that is expressed at very low levels in healthy cucumber plants. PR1 was up-regulated significantly in HSVd-infected plants at 14 and 28 dpi (Figure 6, Table S3), indicating that SA may accumulate to higher levels in HSVd-infected cucumber plants as a result of viroid infection. Our results are consistent with

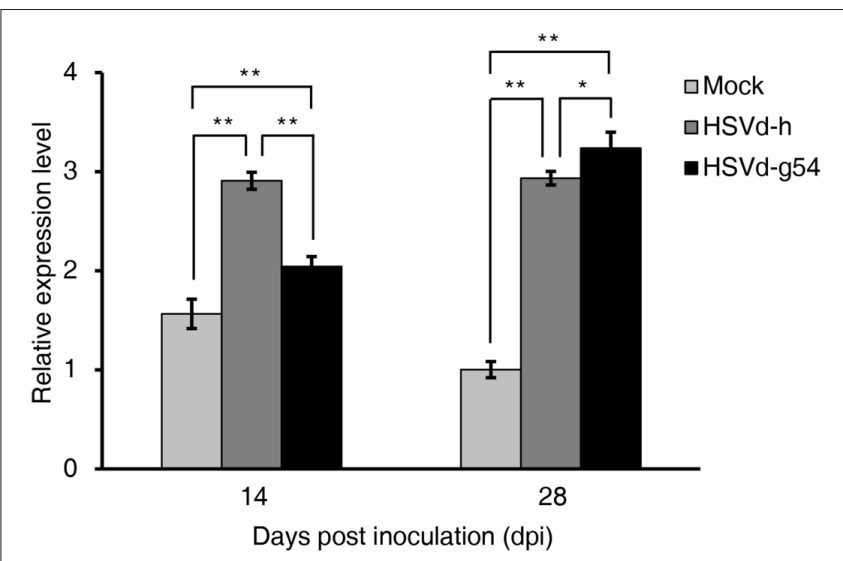

FIGURE 6 | Effect of HSVd infection on PR1 gene expression. Experiments were performed in triplicate and the data are expressed as the mean \pm standard deviation. Error bars indicate the standard deviations of three biological replicates of RT-qPCR analysis. Single or double asterisks indicate statistically significant differences compared with the control at $P<0.05$ or 0.01 , respectively, in Student's $t$-test. EF1 $\alpha$ was used as the reference gene to normalize the gene expression.

those of previous studies. For example, Zheng and colleagues found that genes involved in SA biogenesis and responses, auxin responses, and ethylene biogenesis and responses were induced in PSTVd-infected tomato plants (Zheng et al., 2017).

SA could enhance the resistance of plants to viroid or virus infection. SA activated plant defenses to CEVd and tomato mosaic virus (ToMV) in tomato (Bellés et al., 1999). Plum pox virus (PPV) was able to move to upper non-inoculated leaves of tobacco plants expressing bacterial salicylate hydroxylase (NahG) that degrades SA. Accumulation of virus-derived small RNAs was reduced in the NahG transgenic plants, suggesting that SA might act as an enhancer of the RNA silencing antiviral defense in tobacco (Alamillo et al., 2006). SA treatment induced resistance to CEVd and ToMV in tomato and Gynura auriantiaca, and SA accumulation occured in plants infected by CEVd and ToMV. The RNA silencing-related genes ToDCL1, ToDCL2, ToRDR1, and ToRDR2 were significantly induced by both SA and CEVd in tomato, whereas ToDCL4 and ToRDR6 were only induced by CEVd (Campos et al., 2014). Therefore, both RNA-silencing and SA-mediated defense mechanism could act together to limit viroid and virus infection. Comparing with the corresponding parental plants, the lack of SA accumulation in the CEVdor TSWV-infected NahG transgenic tomato plants led to early symptoms, thus showing that SA is an important component of basal resistance of tomato plants to CEVd and TSWV (LópezGresa et al., 2016). Silencing of SA biosynthetic and signaling genes in Nicotiana benthamiana plants increased susceptibility to tobacco mosaic virus (TMV), indicating that SA was required for systemic resistance response against this virus (Zhu et al., 2014). Therefore, SA may activate plant defenses to viroid and virus infection.

In addition, in the GO analysis of DEGs in HSVdinfected vs. mock-inoculated cucumber plants, we analyzed the top 20 up-regulated GO terms at all three time points 
TABLE 4 | Relative expression of cucumber RDR1 genes in HSVd infected plants.

\begin{tabular}{|c|c|c|c|c|c|}
\hline \multirow[t]{2}{*}{ Pathogen } & \multirow[t]{2}{*}{ Time (dpi) } & \multirow[t]{2}{*}{ Gene name } & \multirow[t]{2}{*}{ Gene ID } & \multicolumn{2}{|c|}{$\log _{2}$ FoldChange } \\
\hline & & & & RNA-seq & RT-qPCR \\
\hline \multirow[t]{5}{*}{ HSVd-h } & 14 & $R D R 1 c 2$ & LOC101213473 & 4.03 & 4.20 \\
\hline & 28 & $R D R 1 c 2$ & LOC101213473 & 4.79 & 15.86 \\
\hline & & $R D R 1 c 1$ & LOC101205704 & 3.44 & 51.51 \\
\hline & & $R D R 1 b$ & LOC101206946 & 1.13 & 3.55 \\
\hline & & RDR1a & LOC101206705 & 0.65 & 1.67 \\
\hline \multirow[t]{5}{*}{ HSVd-g54 } & 14 & $R D R 1 c 2$ & LOC101213473 & 5.17 & 20.60 \\
\hline & & $R D R 1 b$ & LOC101206946 & 1.46 & 3.35 \\
\hline & 28 & $R D R 1 c 2$ & LOC101213473 & 4.58 & 19.06 \\
\hline & & $R D R 1 c 1$ & LOC101205704 & 3.45 & 33.87 \\
\hline & & $R D R 1 b$ & LOC101206946 & 1.25 & 4.03 \\
\hline
\end{tabular}

and found that about 50\% of the top 20 GO terms were related to protein kinase activity, protein phosphorylation, phosphotransferase activity, and transferase activity (transferring phosphorus-containing groups) (Table 1). This observation is consistent with previous reports indicating that PKV (protein kinase-viroid induced) is activated in tomato plants infected with PSTVd (Hammond and Zhao, 2000, 2009). Furthermore, PKV may regulate plant development by functioning in critical signaling pathways involved in gibberellic acid metabolism (Hammond and Zhao, 2009).

\section{Response of CsRDR1 Gene to Viroid Infection}

$R D R 1$ plays an important role in the host response to biotic and abiotic stresses. It is induced by both virus infection (Liu et al., 2009; Leibman et al., 2017) and SA treatment (Xie et al., 2001; Wassenegger and Krczal, 2006; Liu et al., 2009). Thus, it was not unexpected to find that HSVd infection also induces RDR1 expression. CsRDR $1 a$ and $C s R D R 1 b$ expression was only weakly induced at one or two time points; CsRDR1c1, in contrast, was strongly induced by both HSVd-h and HSVd-g54 at $28 \mathrm{dpi}$, and CsRDR1c2 was highly induced by both variants at 14 and $28 \mathrm{dpi}$ (Table 4).

We validated the results of RNA-seq analysis of CsRDR1 gene expression by RT-qPCR. Even though the values obtained by the two methods were different, the data revealed similar trends in the level of CsRDR1 gene expression (Table 4). Several factors (e.g., gene length, GC content, the method of constructing the library for RNA-seq, and the algorithm used to measure gene expression) can affect results obtained by RNAseq (Hansen et al., 2010; Zheng et al., 2011; SEQC/MAQCIII Consortium, 2014; Robert and Watson, 2015; Love et al., 2016), and low expression levels of CsRDR1c1 and CsRDR1c2 in mock-inoculated cucumber plants (Leibman et al., 2017) may cause the different results of the two methods. The induction of RDR1 gene expression in response to HSVd infection observed in our experiments is consistent with that previously found in PSTVd-infected tomato (Schiebel et al., 1998), suggesting that CsRDR1 may play an important role in viroid-host interaction.

Early studies presenting evidence for RNA silencing as a mediator of host-viroid interaction have been reviewed by Ding (2009), and RDR1 is a major component of RNA silencing pathways (Ahlquist, 2002) contributing to basal resistance to several viruses through its role in the biogenesis of virusderived secondary siRNAs (Diaz-Pendon et al., 2007; Qi et al., 2009; Garcia-Ruiz et al., 2010; Wang et al., 2010b). RDR1 is also responsible for production of a distinct class of virusactivated siRNAs (vasiRNAs), which direct widespread silencing of host genes and confer broad-spectrum antiviral activity in Arabidopsis (Cao et al., 2014). Taken together, these studies indicate that $R D R 1$ plays key role in antiviral resistance by silencing both viral RNAs and host immunity-related genes.

In Arabidopsis RNA silencing-mediated viral immunity requires $R D R 1$ and $R D R 6$ to amplify virus-derived siRNAs (Wang et al., 2010b). Previous studies have reported RDR1 orthologs to be virus-, viroid-, or SA-inducible in different plants (Schiebel et al., 1998; Xie et al., 2001; Yang et al., 2004; Alamillo et al., 2006; He et al., 2010; Leibman et al., 2017); furthermore, a correlation between SA-mediated and RNA-silencing defenses has been suggested (Xie et al., 2001). In this study, HSVd infection was shown to induce some genes involved in the SA signaling pathway as well as CsRDR1. To determine whether or not the induction of CsRDR1 by HSVd infection is directly mediated by SA will require additional research. Besides RDR1, some studies have reported that RDR2 (Campos et al., 2014) and RDR6 (Gómez et al., 2008; Di Serio et al., 2010) are also involved in viroid-host interactions. Our studies failed to find any evidence for changes in RDR2 or RDR6 transcription during HSVd infection in cucumber, but these differences may be due to the use of different viroids and host plant species.

In conclusion, we have investigated the global mRNA transcriptional profiles for cucumber plants infected with either a mild (HSVd-h) or a severe (HSVd-g54) variant of HSVd and compared those profiles to the profile in uninfected plants. Identification of DEGs in the present study shows that HSVd-g54 induces an earlier and stronger response than HSVd-h. HSVd infection triggers basal defense responses, depresses photosynthesis, disrupts phytohormone homeostasis, may induce the accumulation and signal transduction of SA, and induces the expression of CsRDR1 genes, especially $C s R D R 1 c 1$ and $C s R D R 1 c 2$, in cucumber plants. Our findings will accelerate research on the interaction between plants and viroids and contribute to a better understanding of the mechanisms that determine viroid pathogenicity.

\section{DATA DEPOSITION}

The raw RNA-seq data were deposited in the NCBI Sequence Read Archive (SRA), and the Accession number is SRR6122525. 


\section{AUTHOR CONTRIBUTIONS}

CX: conducted lab experiments, analyzed the data, and drafted the manuscript; ZZ, TS, and SL: participated in design of the experiments, analyzed the data, and revised the manuscript; $\mathrm{WH}$, ZF, HX, and ML: revised the manuscript. All authors read and approved the final manuscript.

\section{ACKNOWLEDGMENTS}

We are grateful to Professor Robert A. Owens for his help with the writing and his valuable comments. This work was supported by the National Natural Science Foundation of China [grant numbers 31401714 and 31670149].

\section{SUPPLEMENTARY MATERIAL}

The Supplementary Material for this article can be found online at: https://www.frontiersin.org/articles/10.3389/fmicb. 2017.02427/full\#supplementary-material

Figure S1 | Original images of the Northern-blot and the agarose gel before transfer. (A) Original image of the Northern-blot. (B) Original image of the agarose gel before transfer. M, mock; h, cucumber infected with HSVd-h; g54, cucumber

\section{REFERENCES}

Adkar-Purushothama, C., Zhang, Z., Li, S., and Sano, T. (2015). "Analysis and application of viroid-specific small RNAs generated by viroid-inducing RNA silencing," in Plant Virology Protocols: New Approaches to Detect Viruses and Host Responses, eds I. Uyeda and C. Masuta (New York, NY: Humana Press, Springer), 135-170.

Ahlquist, P. (2002). RNA-dependent RNA polymerases, viruses, and RNA silencing. Science 296, 1270-1273. doi: 10.1126/science.1069132

Alamillo, J. M., Saénz, P., and García, J. A. (2006). Salicylic acid-mediated and RNA-silencing defense mechanisms cooperate in the restriction of systemic spread of plum pox virus in tobacco. Plant J. 48, 217-227. doi: $10.1111 / \mathrm{j} .1365-313 X .2006 .02861 . \mathrm{x}$

Ali, R., Ma, W., Lemtiri-Chlieh, F., Tsaltas, D., Leng, Q., von Bodman, S., et al. (2007). Death don't have no mercy and neither does calcium: Arabidopsis CYCLIC NUCLEOTIDE GATED CHANNEL2 and innate immunity. Plant Cell 19, 1081-1095. doi: 10.1105/tpc.106.045096

Allie, F., Pierce, E. J., Okoniewski, M. J., and Rey, C. (2014). Transcriptional analysis of South African cassava mosaic virus-infected susceptible and tolerant landraces of cassava highlights differences in resistance, basal defense and cell wall associated genes during infection. BMC Genomics 15:1006. doi: 10.1186/1471-2164-15-1006

Anders, S., and Huber, W. (2010). Differential expression analysis for sequence count data. Genome Biol. 11:R106. doi: 10.1186/gb-2010-11-10-r106

Anders, S., and Huber, W. (2013). Differential expression of RNA-Seq data at the gene level-the DESeq package. Available online at: http://www.genomatix.de/ online_help/help_regionminer/DESeq_1.10.1.pdf (Accessed on June 23, 2016).

Babu, M., Griffiths, J. S., Huang, T. S., and Wang, A. (2008). Altered gene expression changes in Arabidopsis leaf tissues and protoplasts in response to plum pox virus infection. BMC Genomics 9:325. doi: 10.1186/1471-2164-9-325

Bellés, J. M., Garro, R., Fayos, J., Navarro, P., Primo, J., and Conejero, V. (1999). Gentisic acid as a pathogen-inducible signal, additional to salicylic acid for activation of plant defenses in tomato. Mol. Plant-Microbe. Interact. 12, 227-235. doi: 10.1094/MPMI.1999.12.3.227

Benjamini, Y., and Hochberg, Y. (1995). Controlling the false discovery rate: a practical and powerful approach to multiple testing. J. R. Stat. Soc. Series B Methodol. 57, 289-300. infected with HSVd-g54; PC, positive control (plasmid containing HSVd cDNA dimer).

Figure S2 | Correlation between individual samples (mock, HSVd-h, and HSVd-g54 infection; 2, 14, and 28 dpi) and the comparison of gene expression levels among samples. (A) Pearson correlation between samples. An $R^{2}$ value close to 1 indicates a high degree of correlation between samples. (B) FPKM distribution. FPKM: expected number of Fragments Per Kilobase of transcript sequence per Million base pairs sequenced. M, mock; h, cucumber with HSVd-h infection; g54, cucumber with HSVd-g54 infection.

Table S1 | Oligonucleotide primers used for RT-qPCR analyses.

Table S2 | Statistics of RNA sequencing reads, data quality, Q-scores, and clean reads mapping to the reference genome for nine independent samples isolated from HSVd-infected cucumber plants.

Table S3 | Complete list of DEGs detected in this study.

Table S4 | Validation of RNA-seq results by RT-qPCR.

Table S5 | List of GO terms describing DEGs identified in this study.

Table S6 | List of KEGG pathways containing DEGs identified in this study.

Table S7 | Effect of HSVd infection on the expression of cucumber genes involved in the basal defense response.

Table S8 | KEGG pathway analysis of plant hormone signal transduction in HSVd-infected cucumber. Results for HSVd-h vs. mock, HSVd-g54 vs. mock, and HSVd-g54 vs. HSVd-h comparisons are shown.

Bhattacharyya, D., Gnanasekaran, P., Kumar, R. K., Kushwaha, N. K., Sharma, V. K., Yusuf, M. A., et al. (2015). A geminivirus betasatellite damages the structural and functional integrity of chloroplasts leading to symptom formation and inhibition of photosynthesis. J. Exp. Bot. 66, 5881-5895. doi: 10.1093/jxb/erv299

Blomster, T., Salojärvi, J., Sipari, N., Brosché, M., Ahlfors, R., Keinänen, M., et al. (2011). Apoplastic reactive oxygen species transiently decrease auxin signaling and cause stress-induced morphogenic response in Arabidopsis. Plant Physiol. 157, 1866-1883. doi: 10.1104/pp.111.181883

Boyko, A., Kathiria, P., Zemp, F. J., Yao, Y., Pogribny, I., and Kovalchuk, I. (2007). Transgenerational changes in the genome stability and methylation in pathogen-infected plants: (virus-induced plant genome instability). Nucleic Acids Res. 35, 1714-1725. doi: 10.1093/nar/gkm029

Campos, L., Granell, P., Tárraga, S., López-Gresa, P., Conejero, V., Bellés, J. M., et al. (2014). Salicylic acid and gentisic acid induce RNA silencing-related genes and plant resistance to RNA pathogens. Plant Physiol. Biochem. 77, 35-43. doi: 10.1016/j.plaphy.2014.01.016

Cao, M., Du, P., Wang, X., Yu, Y. Q., Qiu, Y. H., Li, W., et al. (2014). Virus infection triggers widespread silencing of host genes by a distinct class of endogenous siRNAs in Arabidopsis. Proc. Natl. Acad. Sci. U.S.A. 111, 14613-14618. doi: 10.1073/pnas.1407131111

Chen, S., Ye, T., Hao, L., Chen, H., Wang, S., Fan, Z., et al. (2014). Infection of apple by apple stem grooving virus leads to extensive alterations in gene expression patterns but no disease symptoms. PLoS ONE 9:e95239. doi: 10.1371/journal.pone.0095239

Collum, T. D., and Culver, J. N. (2016). The impact of phytohormones on virus infection and disease. Curr. Opin. Virol. 17, 25-31. doi: 10.1016/j.coviro.2015.11.003

Conejero, V., Bellés, J. M., García-Breijo, F., Garro, R., Hernández-Yago, J., Rodrigo, I., et al. (1990). "Signalling in viroid pathogenesis," in Recognition and Response in Plant-Virus Interactions, ed R. S. S. Fraser (Berlin; Heidelberg: Springer), 233-261.

da Graça, J. V., and Martin, M. M. (1981). Ultrastructural changes in avocado leaf tissue infected with avocado sunblotch. J. Phytopathol. 102, 185-194. doi: 10.1111/j.1439-0434.1981.tb03379.x

Daròs, J. A. (2016). "Viroids: small noncoding infectious RNAs with the remarkable ability of autonomous replication," in Current Research Topics in 
Plant Virology, eds A. M. Wang and X. P. Zhou (Cham: Springer International Publishing), 295-322.

Diaz-Pendon, J. A., Li, F., Li, W. X., and Ding, S. W. (2007). Suppression of antiviral silencing by cucumber mosaic virus $2 \mathrm{~b}$ protein in Arabidopsis is associated with drastically reduced accumulation of three classes of viral small interfering RNAs. Plant Cell 19, 2053-2063. doi: 10.1105/tpc.106.047449

Ding, B. (2009). The biology of viroid-host interactions. Annu. Rev. Phytopathol. 47, 105-131. doi: 10.1146/annurev-phyto-080508-081927

Di Serio, F., Martínez de Alba, A. E., Navarro, B., Gisel, A., and Flores, R. (2010). RNA-dependent RNA polymerase 6 delays accumulation and precludes meristem invasion of a viroid that replicates in the nucleus. J. Virol. 84, 2477-2489. doi: 10.1128/JVI.02336-09

Divi, U. K., Rahman, T., and Krishna, P. (2010). Brassinosteroid-mediated stress tolerance in Arabidopsis shows interactions with abscisic acid, ethylene and salicylic acid pathways. BMC Plant Biol. 10:151. doi: 10.1186/1471-2229$10-151$

Fagard, M., Dellagi, A., Roux, C., Périno, C., Rigault, M., Boucher, V., et al. (2007). Arabidopsis thaliana expresses multiple lines of defense to counterattack Erwinia chrysanthemi. Mol. Plant-Microbe Interact. 20, 794-805. doi: 10.1094/MPMI-20-7-0794

Flores, R., Hernández, C., Martínez de Alba, A. E., Daròs, J. A., and Di Serio, F. (2005). Viroids and viroid-host interactions. Annu. Rev. Phytopathol. 43, 117-139. doi: 10.1146/annurev.phyto.43.040204.140243

Flores, R., Minoia, S., Carbonell, A., Gisel, A., Delgado, S., López-Carrasco, A., et al. (2015). Viroids, the simplest RNA replicons: how they manipulate their hosts for being propagated and how their hosts react for containing the infection. Virus Res. 209, 136-145. doi: 10.1016/j.virusres.2015.02.027

Ford, K. A., Casida, J. E., Chandran, D., Gulevich, A. G., Okrent, R. A., Durkin, K. A., et al. (2010). Neonicotinoid insecticides induce salicylate-associated plant defense responses. Proc. Natl. Acad. Sci. U.S.A. 107, 17527-17532. doi: 10.1073/pnas.1013020107

Gago-Zachert, S. (2016). Viroids, infectious long non-coding RNAs with autonomous replication. Virus Res. 212, 12-24. doi: 10.1016/j.virusres. 2015.08.018

Gao, X. Q., Chen, X., Lin, W. W., Chen, S. X., Lu, D. P., Niu, Y. J., et al. (2013). Bifurcation of Arabidopsis NLR immune signaling via $\mathrm{Ca}^{2+}$-dependent protein kinases. PLoS Pathog. 9:e1003127. doi: 10.1371/journal.ppat.1003127

Garcia-Ruiz, H., Takeda, A., Chapman, E. J., Sullivan, C. M., Fahlgren, N., Brempelis, K. J., et al. (2010). Arabidopsis RNA-dependent RNA polymerases and Dicer-like proteins in antiviral defense and small interfering RNA biogenesis during turnip mosaic virus infection. Plant Cell 22, 481-496. doi: 10.1105/tpc.109.073056

Gómez, G., Martínez, G., and Pallás, V. (2008). Viroid-induced symptoms in Nicotiana benthamiana plants are dependent on RDR6 activity. Plant Physiol. 148, 414-423. doi: 10.1104/pp.108.120808

Gómez-Gómez, L., and Boller, T. (2000). FLS2: an LRR receptor-like kinase involved in the perception of the bacterial elicitor flagellin in Arabidopsis. Mol. Cell 5, 1003-1011. doi: 10.1016/S1097-2765(00)80265-8

Hammond, R. W., and Zhao, Y. (2000). Characterization of a tomato protein kinase gene induced by infection by potato spindle tuber viroid. Mol. PlantMicrobe Interact. 13, 903-910. doi: 10.1094/MPMI.2000.13.9.903

Hammond, R. W., and Zhao, Y. (2009). Modification of tobacco plant development by sense and antisense expression of the tomato viroid-induced AGC VIIIa protein kinase PKV suggests involvement in gibberellin signaling. BMC Plant Biol. 9:108. doi: 10.1186/1471-2229-9-108

Hansen, K. D., Brenner, S. E., and Dudoit, S. (2010). Biases in Illumina transcriptome sequencing caused by random hexamer priming. Nucleic Acids Res. 38:e131. doi: 10.1093/nar/gkq224

Hari, V. (1980). Ultrastructure of potato spindle tuber viroid-infected tomato leaf tissue. Phytopathology 70, 385-387. doi: 10.1094/Phyto-70-385

He, J., Dong, Z., Jia, Z., Wang, J., and Wang, G. (2010). Isolation, expression and functional analysis of a putative RNA-dependent RNA polymerase gene from maize (Zea mays L.). Mol. Biol. Rep. 37, 865-874. doi: $10.1007 / \mathrm{s} 11033-009-9692-2$

Huang, S., Li, R., Zhang, Z., Li, L., Gu, X., Fan, W., et al. (2009). The genome of the cucumber, Cucumis sativus L. Nat. Genet. 41, 1275-1281. doi: 10.1038/ng.475

Itaya, A., Matsuda, Y., Gonzales, R. A., Nelson, R. S., and Ding, B. (2002). Potato spindle tuber viroid strains of different pathogenicity induces and suppresses expression of common and unique genes in infected tomato. Mol. Plant-Microbe Interact. 15, 990-999. doi: 10.1094/MPMI.2002.15.10.990

Kappagantu, M., Bullock, J. M., Nelson, M. E., and Eastwell, K. C. (2017). Hop stunt viroid: effect on host (Humulus lupulus) transcriptome and its interactions with hop powdery mildew (Podspheara macularis). Mol. Plant-Microbe Interact. 30, 842-851. doi: 10.1094/MPMI-03-17-0071-R

Kawaguchi-Ito, Y., Li, S. F., Tagawa, M., Araki, H., Goshono, M., Yamamoto, S., et al. (2009). Cultivated grapevines represent a symptomless reservoir for the transmission of hop stunt viroid to hop crops: 15 years of evolutionary analysis. PLOS ONE 4:e8386. doi: 10.1371/journal.pone.00 08386

Kojima, M., Murai, M., and Shikata, E. (1983). Cytopathic changes in viroidinfected leaf tissues. J. Fac. Agric. Hokkaido Univ. 61, 219-223.

Kuzniak, E., Wielanek, M., Chwatko, G., Głowacki, R., Libik-Konieczny, M., Piatek, M., et al. (2015). Salicylic acid and cysteine contribute to arbutininduced alleviation of angular leaf spot disease development in cucumber. $J$. Plant Physiol. 181, 9-13. doi: 10.1016/j.jplph.2015.03.017

Leibman, D., Kravchik, M., Wolf, D., Haviv, S., Weissberg, M., Ophir, R., et al. (2017). Differential expression of cucumber RNA-dependent RNA polymerase 1 genes during antiviral defence and resistance. Mol. Plant Pathol. doi: 10.1111/mpp.12518. [Epub ahead of print].

Li, Y., Cui, H., Cui, X., and Wang, A. (2016). The altered photosynthetic machinery during compatible virus infection. Curr. Opin. Virol. 17, 19-24. doi: 10.1016/j.coviro.2015.11.002

Linthorst, H. J., and Van Loon, L. (1991). Pathogenesis-related proteins of plants. Crit. Rev. Plant Sci. 10, 123-150. doi: 10.1080/07352689109382309

Liu, Y., Gao, Q., Wu, B., Ai, T., and Guo, X. (2009). NgRDR1, an RNA-dependent RNA polymerase isolated from Nicotiana glutinosa, was involved in biotic and abiotic stresses. Plant Physiol. Biochem. 47, 359-368. doi: 10.1016/j.plaphy.2008.12.017

López-Gresa, M. P., Lisón, P., Yenush, L., Conejero, V., Rodrigo, I., and Bellés, J. M. (2016). Salicylic acid is involved in the basal resistance of tomato plants to citrus exocortis viroid and tomato spotted wilt virus. PLoS ONE 11:e0166938. doi: 10.1371/journal.pone. 0166938

Love, M. I., Hogenesch, J. B., and Irizarry, R. A. (2016). Modeling of RNAseq fragment sequence bias reduces systematic errors in transcript abundance estimation. Nat. Biotechnol. 34:1287. doi: 10.1038/nbt.3682

Mao, X., Cai, T., Olyarchuk, J. G., and Wei, L. P. (2005). Automated genome annotation and pathway identification using the KEGG Orthology (KO) as a controlled vocabulary. Bioinformatics 21, 3787-3793. doi: 10.1093/bioinformatics/bti430

Momma, T., and Takahashi, T. (1983). Cytopathology of shoot apical meristem of hop plants infected with hop stunt viroid. J. Phytopathol. 106, 272-280. doi: 10.1111/j.1439-0434.1983.tb00052.x

Navarro, B., Gisel, A., Rodio, M. E., Delgado, S., Flores, R., and Di Serio, F. (2012). Small RNAs containing the pathogenic determinant of a chloroplast-replicating viroid guide the degradation of a host mRNA as predicted by RNA silencing. Plant J. 70, 991-1003. doi: 10.1111/j.1365-313X.2012.04940.x

Otulak, K., Chouda, M., Bujarski, J., and Garbaczewska, G. (2015). The evidence of tobacco rattle virus impact on host plant organelles ultrastructure. Micron 70 , 7-20. doi: 10.1016/j.micron.2014.11.007

Owens, R. A., Tech, K. B., Shao, J. Y., Sano, T., and Baker, C. J. (2012). Global analysis of tomato gene expression during potato spindle tuber viroid infection reveals a complex array of changes affecting hormone signaling. Mol. Plant-Microbe Interact. 25, 582-598. doi: 10.1094/MPMI-0911-0258

Ozsolak, F., and Milos, P. M. (2011). RNA sequencing: advances, challenges and opportunities. Nat. Rev. Genet. 12, 87-98. doi: 10.1038/nrg2934

Pallas, V., and García, J. (2011). How do plant viruses induce disease? Interactions and interference with host components. J. Gen. Virol. 92, 2691-2705. doi: 10.1099/vir.0.034603-0

Pérez-Clemente, R. M., Montoliu, A., Vives, V., López-Climent, M. F., and GómezCadenas, A. (2015). Photosynthetic and antioxidant responses of Mexican lime (Citrus aurantifolia) plants to citrus tristeza virus infection. Plant Pathol. 64, 16-24. doi: 10.1111/ppa.12241

Pokorn, T., Radišek, S., Javornik, B., Štajner, N., and Jakše, J. (2017). Development of hop transcriptome to support research into host-viroid interactions. PLoS ONE 12:e0184528. doi: 10.1371/journal.pone.0184528 
Prod'homme, D., Le Panse, S., Drugeon, G., and Jupin, I. (2001). Detection and subcellular localization of the Turnip yellow mosaic virus $66 \mathrm{~K}$ replication protein in infected cells. Virology 281, 88-101. doi: 10.1006/viro.2000.0769

Qi, X., Bao, F. S., and Xie, Z. (2009). Small RNA deep sequencing reveals role for Arabidopsis thaliana RNA-dependent RNA polymerases in viral siRNA biogenesis. PLoS ONE 4:e4971. doi: 10.1371/journal.pone.0004971

Rizza, S., Conesa, A., Juarez, J., Catara, A., Navarro, L., Duran-Vila, N., et al. (2012). Microarray analysis of Etrog citron (Citrus medica L.) reveals changes in chloroplast, cell wall, peroxidase and symporter activities in response to viroid infection. Mol. Plant Pathol. 13, 852-864. doi: 10.1111/j.1364-3703.2012.00794.x

Robert, C., and Watson, M. (2015). Errors in RNA-Seq quantification affect genes of relevance to human disease. Genome Biol. 16:177. doi: 10.1186/s13059-015-0734-x

Rodio, M. E., Delgado, S., De Stradis, A., Gómez, M. D., Flores, R., and Di Serio, F. (2007). A viroid RNA with a specific structural motif inhibits chloroplast development. Plant Cell 19, 3610-3626. doi: 10.1105/tpc.106.049775

Rodriguez, M. C., Conti, G., Zavallo, D., Manacorda, C. A., and Asurmendi, S. (2014). TMV-Cg Coat Protein stabilizes DELLA proteins and in turn negatively modulates salicylic acid-mediated defense pathway during Arabidopsis thaliana viral infection. BMC Plant Biol. 14:210. doi: 10.1186/s12870-014-0210-x

Sampol, B., Bota, J., Riera, D., Medrano, H., and Flexas, J. (2003). Analysis of the virus-induced inhibition of photosynthesis in malmsey grapevines. New Phytol. 160, 403-412. doi: 10.1046/j.1469-8137.2003.00882.x

Sano, T., Mimura, R., and Ohshima, K. (2001). Phylogenetic analysis of hop and grapevine isolates of hop stunt viroid supports a grapevine origin for hop stunt disease. Virus Genes. 22, 53-59. doi: 10.1023/A:1008182302704

Schiebel, W., Pélissier, T., Riedel, L., Thalmeir, S., Schiebel, R., Kempe, D., et al. (1998). Isolation of an RNA-Directed RNA polymerase-specific cDNA clone from tomato. Plant Cell 10, 2087-2101.

SEQC/MAQC-III Consortium (2014). A comprehensive assessment of RNA-seq accuracy, reproducibility and information content by the Sequencing Quality Control Consortium. Nat. Biotechnol. 32, 903-914. doi: 10.1038/nbt.2957

Tena, G., Boudsocq, M., and Sheen, J. (2011). Protein kinase signaling networks in plant innate immunity. Curr. Opin. Plant Biol. 14, 519-529. doi: 10.1016/j.pbi.2011.05.006

Tessitori, M., Maria, G., Capasso, C., Catara, G., Rizza, S., De Luca, V., et al. (2007). Differential display analysis of gene expression in Etrog citron leaves infected by citrus viroid III. Biochim. Biophys. Acta. 1769, 228-235. doi: 10.1016/j.bbaexp.2007.03.004

Trapnell, C., Williams, B. A., Pertea, G., Mortazavi, A., Kwan, G., van Baren, M. J., et al. (2010). Transcript assembly and quantification by RNA-Seq reveals unannotated transcripts and isoform switching during cell differentiation. Nat. Biotechnol. 28, 511-515. doi: 10.1038/nbt.1621

Van Verk, M. C., Hickman, R., Pieterse, C. M., and Van Wees, S. C. (2013). RNA-Seq: revelation of the messengers. Trends Plant Sci. 18, 175-179. doi: 10.1016/j.tplants.2013.02.001

Wan, H., Zhao, Z., Qian, C., Sui, Y., Malik, A. A., and Chen, J. (2010). Selection of appropriate reference genes for gene expression studies by quantitative realtime polymerase chain reaction in cucumber. Anal. Biochem. 399, 257-261. doi: 10.1016/j.ab.2009.12.008

Wang, L., Feng, Z., Wang, X., Wang, X., and Zhang, X. (2010a). DEGseq: an $R$ package for identifying differentially expressed genes from RNA-seq data. Bioinformatics 26, 136-138. doi: 10.1093/bioinformatics/btp612

Wang, M. B., Masuta, C., Smith, N. A., and Shimura, H. (2012). RNA silencing and plant viral diseases. Mol. Plant-Microbe Interact. 25, 1275-1285. doi: 10.1094/MPMI-04-12-0093-CR

Wang, X. B., Wu, Q. F., Ito, T., Cillo, F., Li, W. X., Chen, X. M., et al. (2010b). RNAi-mediated viral immunity requires amplification of virusderived siRNAs in Arabidopsis thaliana. Proc. Natl. Acad. Sci. U.S.A. 107, 484-489. doi: 10.1073/pnas.0904086107
Wang, Y., Shibuya, M., Taneda, A., Kurauchi, T., Senda, M., Owens, R. A., et al. (2011). Accumulation of potato spindle tuber viroid-specific small RNAs is accompanied by specific changes in gene expression in two tomato cultivars. Virology 413, 72-83. doi: 10.1016/j.virol.2011.01.021

Wang, Z., Gerstein, M., and Snyder, M. (2009). RNA-Seq: a revolutionary tool for transcriptomics. Nat. Rev. Genet. 10, 57-63. doi: 10.1038/nrg2484

Wassenegger, M., and Krczal, G. (2006). Nomenclature and functions of RNA-directed RNA polymerases. Trends Plant Sci. 11, 142-151. doi: 10.1016/j.tplants.2006.01.003

Whenham, R. J., Fraser, R. S., Brown, L. P., and Payne, J. A. (1986). Tobaccomosaic-virus-induced increase in abscisic-acid concentration in tobacco leaves. Planta 168, 592-598. doi: 10.1007/BF00392281

Whitham, S. A., Yang, C., and Goodin, M. M. (2006). Global impact: elucidating plant responses to viral infection. Mol. Plant-Microbe Interact. 19, 1207-1215. doi: 10.1094/MPMI-19-1207

Wu, S., Shan, L., and He, P. (2014). Microbial signature-triggered plant defense responses and early signaling mechanisms. Plant Sci. 228, 118-126. doi: 10.1016/j.plantsci.2014.03.001

Xie, Z., Fan, B., Chen, C., and Chen, Z. (2001). An important role of an inducible RNA-dependent RNA polymerase in plant antiviral defense. Proc. Natl. Acad. Sci. U.S.A. 98, 6516-6521. doi: 10.1073/pnas. 111440998

Yang, S. J., Carter, S. A., Cole, A. B., Cheng, N. H., and Nelson, R. S. (2004). A natural variant of a host RNA-dependent RNA polymerase is associated with increased susceptibility to viruses by Nicotiana benthamiana. Proc. Natl. Acad. Sci. U.S.A. 101, 6297-6302. doi: 10.1073/pnas.0304346101

Young, M. D., Wakefield, M. J., Smyth, G. K., and Oshlack, A. (2010). Gene ontology analysis for RNA-seq: accounting for selection bias. Genome Biol. 11:R14. doi: 10.1186/gb-2010-11-2-r14

Zechmann, B., Müller, M., and Zellnig, G. (2003). Cytological modifications in zucchini yellow mosaic virus (ZYMV)-infected Styrian pumpkin plants. Arch. Virol. 148, 1119-1133. doi: 10.1007/s00705-003-0005-0

Zheng, W., Chung, L. M., and Zhao, H. (2011). Bias detection and correction in RNA-Sequencing data. BMC Bioinformatics 12:290. doi: 10.1186/1471-2105-12-290

Zheng, Y., Wang, Y., Ding, B., and Fei, Z. J. (2017). Comprehensive transcriptome analyses reveal that potato spindle tuber viroid triggers genome-wide changes in alternative splicing, inducible trans-acting activity of phasiRNAs and immune responses. J. Virol. 91:e00247-17. doi: 10.1128/JVI.00247-17

Zhu, F., Xi, D. H., Yuan, S., Xu, F., Zhang, D. W., and Lin, H. H. (2014). Salicylic acid and jasmonic acid are essential for systemic resistance against tobacco mosaic virus in Nicotiana benthamiana. Mol. Plant Microbe Interact. 27, 567-577. doi: 10.1094/MPMI-11-13-0349-R

Zhu, S., Gao, F., Cao, X., Chen, M., Ye, G., Wei, C., et al. (2005). The rice dwarf virus $\mathrm{P} 2$ protein interacts with ent-kaurene oxidases in vivo, leading to reduced biosynthesis of gibberellins and rice dwarf symptoms. Plant Physiol. 139, 1935-1945. doi: 10.1104/pp.105.072306

Conflict of Interest Statement: The authors declare that the research was conducted in the absence of any commercial or financial relationships that could be construed as a potential conflict of interest.

The handling Editor declared a past co-authorship with one of the authors SL and TS.

Copyright (c) 2017 Xia, Li, Hou, Fan, Xiao, Lu, Sano and Zhang. This is an openaccess article distributed under the terms of the Creative Commons Attribution License (CC BY). The use, distribution or reproduction in other forums is permitted, provided the original author(s) or licensor are credited and that the original publication in this journal is cited, in accordance with accepted academic practice. No use, distribution or reproduction is permitted which does not comply with these terms. 